\title{
Neutrophils contribute to spontaneous resolution of liver inflammation and fibrosis via microRNA-223
}

\author{
Carolina Jimenez Calvente, ${ }^{1}$ Masahiko Tameda, ${ }^{1}$ Casey D. Johnson, ${ }^{1}$ Hana del Pilar, ${ }^{1}$ Yun Chin Lin, ${ }^{1}$ Nektaria Adronikou, ${ }^{2}$ \\ Xavier De Mollerat Du Jeu, ${ }^{2}$ Cristina Llorente, ${ }^{3}$ Josh Boyer, ${ }^{3}$ and Ariel E. Feldstein ${ }^{1}$ \\ 'Department of Pediatrics, School of Medicine, UCSD, San Diego, California, USA. ${ }^{2}$ Thermo Fisher Scientific, Carlsbad, California, USA. ${ }^{3}$ Department of Medicine, School of Medicine, UCSD, San Diego, \\ California, USA
}

\begin{abstract}
Persistent, unresolved inflammation in the liver represents a key trigger for hepatic injury and fibrosis in various liver diseases and is controlled by classically activated proinflammatory macrophages, while restorative macrophages of the liver are capable of reversing inflammation once the injury trigger ceases. Here we exhibit neutrophils as key contributors to resolving the inflammatory response in the liver using two models of liver inflammation resolution. Using two models of liver inflammatory resolution, we found that mice undergoing neutrophil depletion during the resolution phase exhibited unresolved hepatic inflammation, activation of the fibrogenic machinery, and early fibrosis. These findings were associated with an impairment of the phenotypic switch of proinflammatory macrophages into a restorative stage after removal of the cause of injury and an increased NLRP3/miR-223 ratio. Mice with a deletion of the granulocyte-specific miR-223 gene showed a similarly impaired resolution profile that could be reversed by replacing miR-223 levels using a miR-223 $3 p$ mimic or by infusion of neutrophils from wild-type animals. Collectively, our findings reveal hepatic neutrophils as resolving effector cells that induce proinflammatory macrophages into a restorative phenotype, potentially via miR-223.
\end{abstract}

\section{Introduction}

Liver inflammation constitutes a central contributor to liver pathology during chronic injury from varying etiologies $(1,2)$. While it is self-limiting, liver inflammation is considered beneficial and contributes to tissue homeostasis (2). The inability to shut down the persistence of the triggers of the inflammatory response results in tissue dysfunction and perpetuates the disease state (3). Inflammation in the liver is typically characterized by de novo recruitment of a repertoire of inflammatory cells mainly composed of monocytes, early activated proinflammatory macrophages, neutrophils, and NK cells that compromise hepatic vasculate architecture and function (4). The systemic response to persistent inflammation is impaired wound healing with subsequent activation of fibrogenic pathways and an overall progression to fibrosis - the excessive accumulation of extracellular matrix (5) that potentially leads to cirrhosis, which sets the stage for hepatic decompensation, primary liver cancer, and death (6). In some instances, the liver can reverse injurious and fibrotic changes with the use of direct-acting antiviral agents directed to treat chronic hepatitis $\mathrm{C}$ virus infection, corticoid therapy in patients with autoimmune hepatitis, cessation of alcohol consumption in patients with alcoholic liver disease, or bariatric surgery to induce significant and sustained weight loss in obese patients with nonalco-

Authorship note: CJC and MT contributed equally to this work.

Conflict of interest: The authors have declared that no conflict of interest exists.

Copyright: @ 2019, American Society for Clinical Investigation.

Submitted: May 14, 2018; Accepted: July 3, 2019; Published: August 26, 2019.

Reference information: J Clin Invest. 2019;129(10):4091-4109.

https://doi.org/10.1172/JCl122258. holic steatohepatitis (NASH) (7-12). Therefore, it is of paramount importance to uncover the mechanism involved in the natural (also called spontaneous) resolution of liver inflammation in order to identify potential novel targets for therapeutic intervention in those patients who do not respond to current therapies or for whom no effective interventions are currently available.

Neutrophils have recently emerged as important contributors to the resolution of the inflammatory response in various tissues, including the heart, skin, and joints (13-17). However, the mechanism by which neutrophils contribute to the resolution of inflammation remains incompletely understood.

MicroRNAs, naturally occurring small RNA molecules, play an important role in the regulation of gene expression involved in a variety of cellular processes, including inflammation. Several microRNAs have been identified as being involved in hepatic pathophysiology. Among them, miR-223, which is preferentially expressed in granulocytes $(18,19)$ and is a critical negative regulator of NLRP3 inflammasome activity $(18,20)$, has been identified as one of the most significantly downregulated microRNAs in the livers of patients with NASH (21), while miR-223-knockout mice are highly sensitive to liver inflammation and injury when exposed to low-dose endotoxin injection (19). In this study we examined the role of neutrophils during the resolution phase of liver inflammation and uncovered miR-223 as a novel antiinflammatory, antifibrotic therapeutic target.

\section{Results}

Neutrophil depletion impairs spontaneous resolution of liver inflammation. To study the role of neutrophils as potential mediators of spontaneous resolution of liver inflammation (SRLI), we 
first established a mouse model of systemic neutrophil depletion during the spontaneous recovery from liver inflammation after cessation of the cause of injury. The mice were gavaged for 1 week with carbon tetrachloride (CCL4) to induce liver inflammation. One group of mice was sacrificed 4 hours after CCL 4 treatment to serve as a putative inflammatory baseline control (called the 4 hours post-CCL 4 group). The rest of the mice were allowed to recover from inflammation naturally for 96 hours following the full-course treatment (1 week) with CCL4. An independent group of these mice were subjected to systemic neutrophil depletion using an i.v. injection of anti-Ly6G mAb (serving as the 96 hours post-CCL4 + anti-Ly6G mAb group) or PBS (representing the 96 hours post-CCL 4 untreated control) at $0.1 \mathrm{mg} / \mathrm{mouse}$ for 3 consecutive days and were sacrificed 24 hours after final injection (end of the 96 hours resolution stage) (Figure 1A). In comparison with an independent group of mice injected with the neutrophil depletion isotype control IgG2b mAb following the same i.v. regimen described above, the treatment with anti-Ly6 G mAb led to almost complete neutralization of circulating neutrophils (Supplemental Figure 1, A and B; supplemental material available online with this article; https://doi.org/10.1172/JCI122258DS1) and significantly increased the serum levels of the liver inflammation surrogate alanine aminotransferase (ALT) during the 96 hours post-CCL4 resolution phase (Figure 1B; vs. non-neutrophil-depleted mice). The livers from mice not treated with anti-Ly6 $\mathrm{G}$ mAb exhibited almost complete recovery with no evidence of necroinflammation at 96 hours after CCL4 administration when compared with the livers of mice sacrificed 4 hours after CCL 4 treatment (Figure 1, C and D); these results corroborate previously published data (22). Furthermore, the neutrophil-depleted livers from anti-Ly6G mAbinjected mice displayed significant necroinflammatory changes (Ishak histology scoring, ref. 23) compared with those from mice that did not receive anti-Ly6G mAb, and similarly to those from mice sacrificed 4 hours after CCL 4 treatment (Figure 1, C and D), indicating a worsening of the liver architecture. In addition, we observed areas within the liver densely occupied by macrophages that stained positive for F4/80 (Figure 1E); similar F4/80-positive liver areas were also confirmed in a different experiment under the same set of conditions but using a different clone of the antiLy6G antibody (Supplemental Figures 2 and 3).

Similarly, the serum levels of the inflammatory cytokine IL-12 sharply increased (vs. untreated controls) (Figure 1F), whereas the specific antiinflammatory cytokine IL-10, a marker of restorative macrophages (24), dramatically decreased (Figure 1G), indicating an impaired systemic inflammatory condition.

To further assess the proinflammatory function of neutrophils in SRLI, we defined a liver macrophage phenotype profile of our experimental groups shown in Figure 1A by staining with antibodies specific for either proinflammatory (Ly6C) or restorative (CD163 and CD14) macrophage markers (24-29). The area stained for Ly6C was significantly expanded in neutrophildepleted mice when observed in relation to those mice that did not undergo neutrophil depletion (Figure 2, A and B). Conversely, the staining for CD163- and CD14-positive macrophages was significantly lower in mice given anti-Ly6G mAb compared with their control animals not treated with anti-Ly6G mAb (Figure 2, $\mathrm{C}$ and D). To further support these data, we determined that treatment with anti-Ly6G mAb resulted in a pronounced upregulation of transcripts of the monocyte chemoattractants Ccl4 and Ccl2 (refs. 30, 31, and Figure 2, E and F), which partly correlated with the hepatic levels of CCL2 and the CCL3 and CCL4 receptor CCR5 (Supplemental Figures 4 and 5).

Taken together, these results illustrate the contribution of neutrophils in SRLI.

Neutrophil deletion results in the persistence of classically activated hepatic macrophages during SRLI. To better understand the mechanisms by which neutrophils participate in SRLI, hepatic macrophages were isolated from our experimental mice (Figure 1A) and confirmed by FACS using F4/80 expression as a selection target (Supplemental Figure 6A; gating strategy shown in Supplemental Figure 7). Next, we performed a gene expression analysis of $i$ Nos, the key enzyme of (classically activated) proinflammatory macrophages, and the restorative macrophage-specific markers Arg1, Il10, and Ager (a scavenger receptor enriched in putative, antiinflammatory macrophages; refs. 32-34). The expression of $i$ Nos mRNA was dramatically downregulated in the hepatic macrophages of mice that were allowed to recover for 4 days compared with the control mice that presented a pronounced inflammation 4 hours after CCL4 (Supplemental Figure 6B). In contrast, the mice that were treated with anti-Ly6 $\mathrm{G}$ mAb during the recovery phase showed robust expression of $i$ Nos mRNA compared with the untreated control mice at 96 hours after CCL4 (Supplemental Figure 6B). Moreover, the group of mice treated with anti-Ly6G mAb displayed significant downregulation of both Arg1 and Il1O when compared with the untreated group (Supplemental Figure 6, C and D). Accordingly, Ager expression was significantly reduced in neutrophil-depleted mice compared with the nondepleted control group at 96 hours after CCL4 (Supplemental Figure 6E), which further supports the antiinflammatory role of neutrophils in the liver.

These results suggest that neutrophils play a pivotal role as negative regulators of classical macrophage activation in SRLI.

Neutrophil abrogation worsens spontaneous resolution of early liver fibrosis. Persistent activation of macrophages as a result of hepatic inflammation typically initiates early fibrogenesis (5), a wound healing response consisting of increased collagen production by activated hepatic stellate cells (HSCs) (5). We examined the potential effect of neutrophils on downstream, persistent inflammation by performing Sirius red and antiaSMA staining in livers of mice from the experiment in Figure $1 \mathrm{~A}$ to determine total collagen accumulation and HSC activation, respectively. We observed negligible signs of collagen accumulation or HSC activation 96 hours after the final scheduled dose of CCL4 as opposed to mice sacrificed 4 hours after CCL 4 treatment (Figure 3, A-C), denoting fibrosis resolution. By contrast, the treatment with anti-Ly6G mAb to deplete neutrophils significantly exacerbated collagen deposition and enhanced HSC activation 96 hours after CCL4 administration in comparison with mice untreated with anti-Ly6G mAb (Figure 3, A-C); these results were replicated in a different experiment under the same conditions but using another clone of the anti-Ly6G antibody (Supplemental Figures 2 and 3).

Likewise, the mRNA expression of the collagen-degrading metalloproteinases $M m p 3$ and $M m p 8$ was significantly downreg- 
A

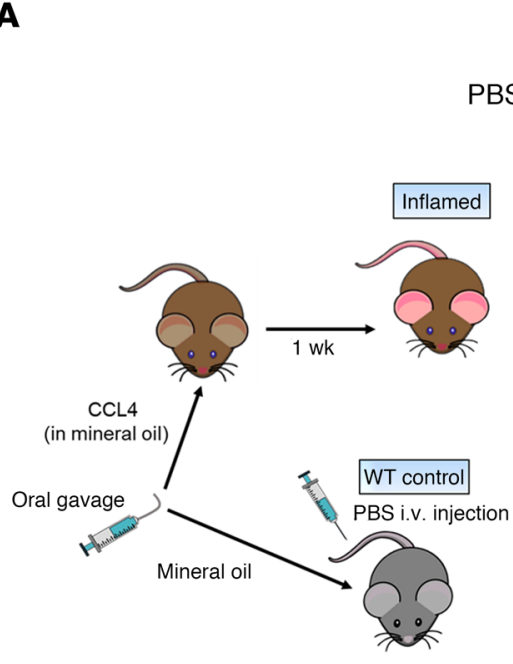

After-CCL4 period

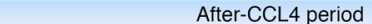

BS i.v. injection
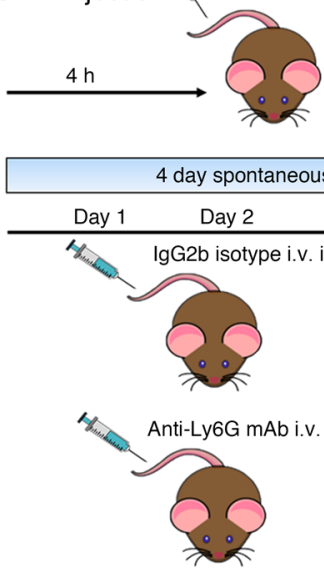

C

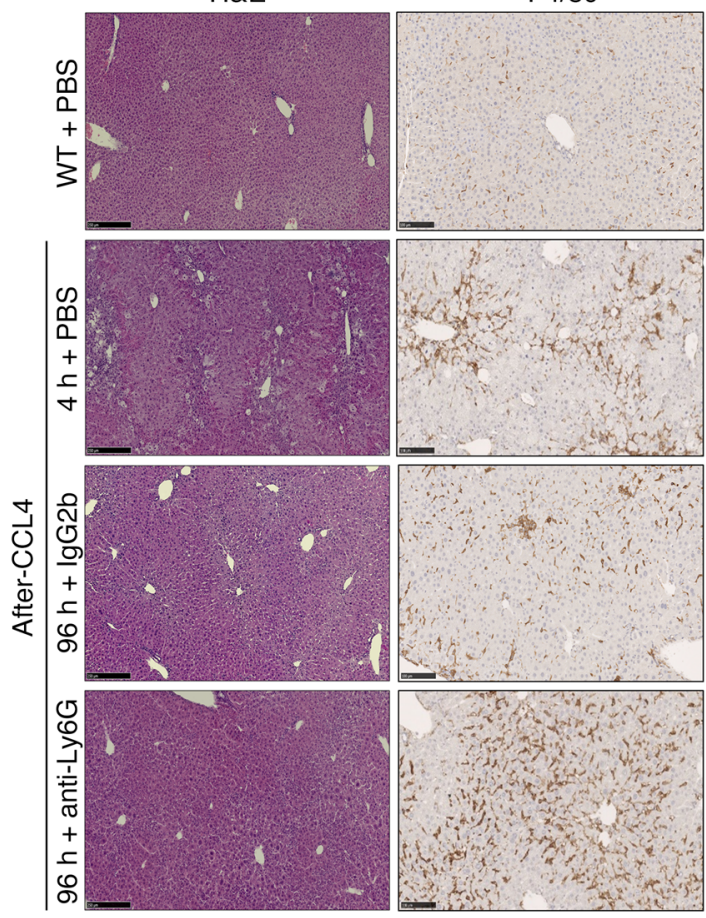

D

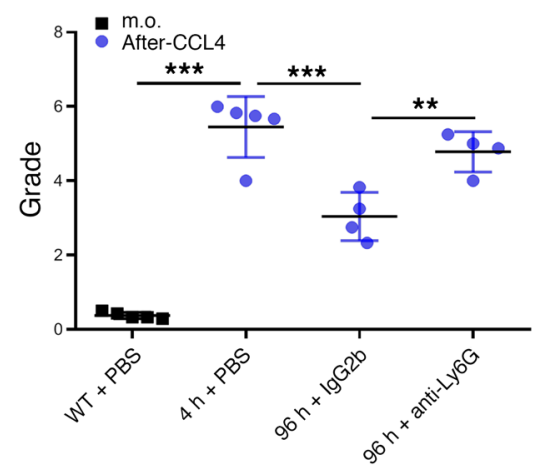

E

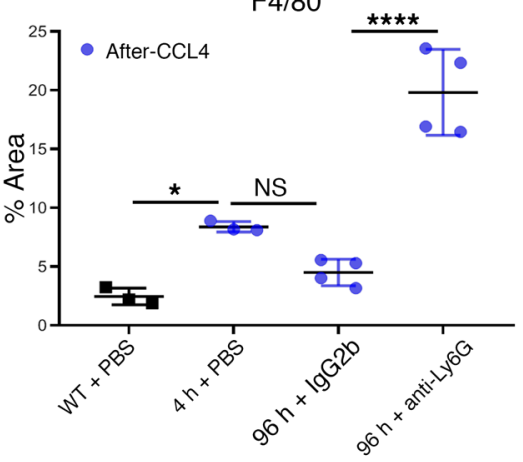

B

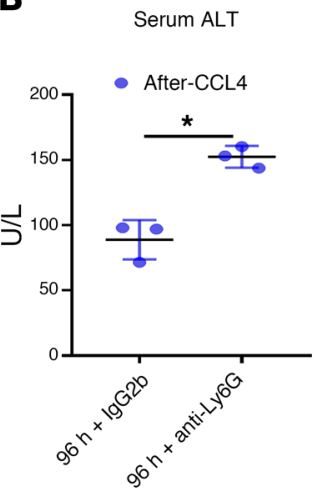

$\mathbf{F}$

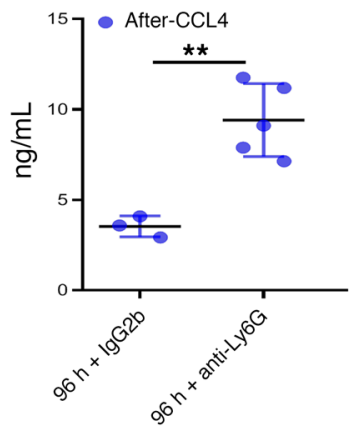

G

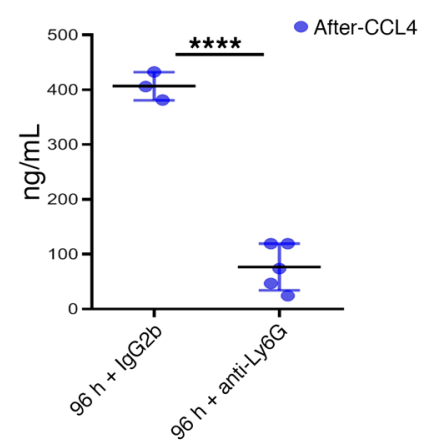

Figure 1. Neutrophil depletion impairs spontaneous resolution of liver inflammation. (A) Schematic representation of the experimental design of the systemic neutrophil depletion model during spontaneous resolution of liver inflammation (SRLI) after treatment with CCL4. (B) Serum ALT levels measured by colorimetry. ${ }^{*} P<0.05$, 2-tailed unpaired $t$ test, $n=3$ per group. (C) Representative images of liver sections stained for parenchymal and nonparenchymal cells and total macrophages with H\&E and antibody to F4/80, respectively. Scale bars: 250 or $100 \mu \mathrm{m}$ for $\mathrm{H} \& \mathrm{E}$ or F4/80, respectively. $n=4-5$ per group. (D) Grade of necroinflammation as assessed by the Ishak system. ${ }^{* *} P<0.01,{ }^{* *} P<0.001,1$-way ANOVA, $n=3-6$ per group. (E) Percentage of F4/80-positive area in 10 randomly selected fields, by computerized image analysis. ${ }^{*} P<0.05,{ }^{* * *} P<0.0001,1$-way ANOVA, $n=3-4$ per group. (F and $\mathbf{G}$ ) Serum levels of IL-12 and IL-10 quantified by colorimetric and luminescent ELISA, respectively, using biotinylated mouse mAbs for IL-12 or IL-10. ${ }^{* *} P<0.01$, ${ }^{* * * *} P<0.0001,2$-tailed unpaired $t$ test. Data are represented as means \pm SD, $n=3-5$ per group. Representative experiment of 2 independent repeats. 
A
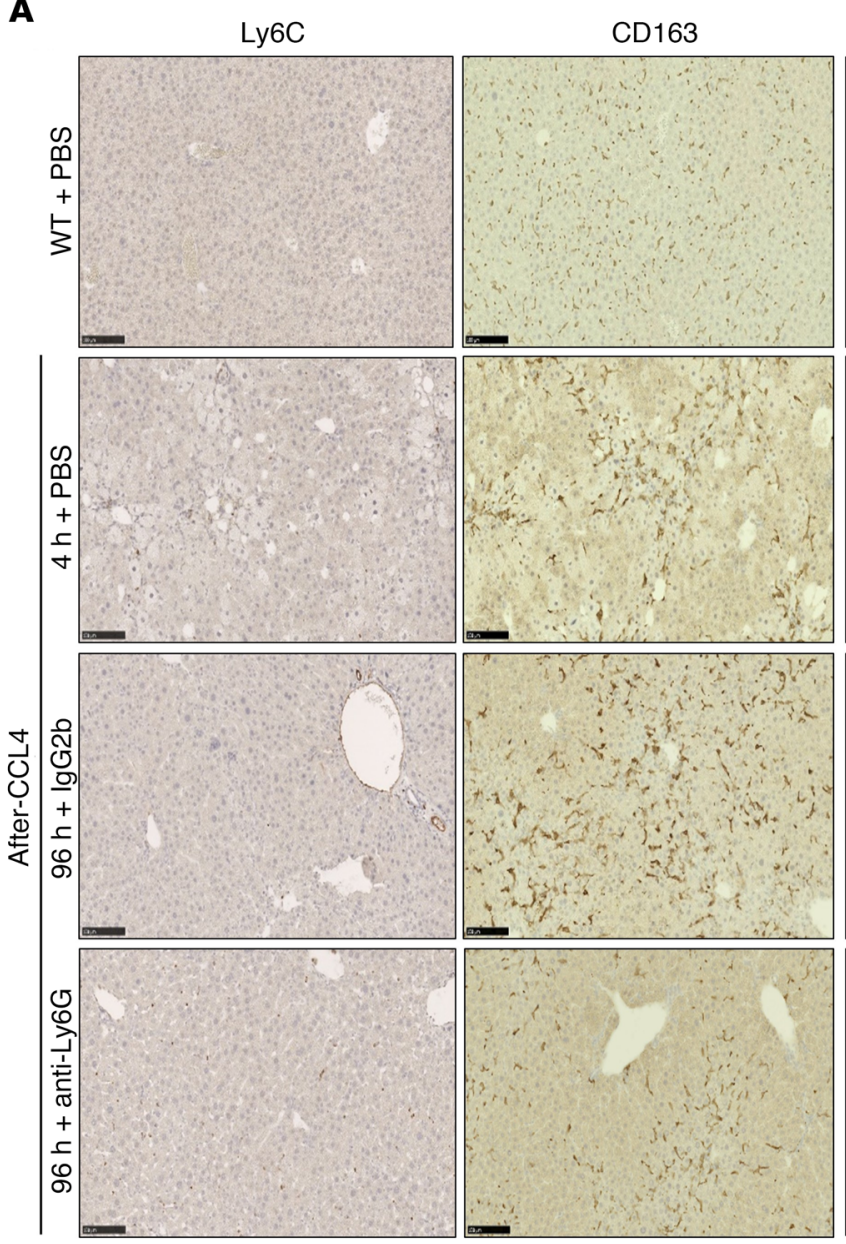

E

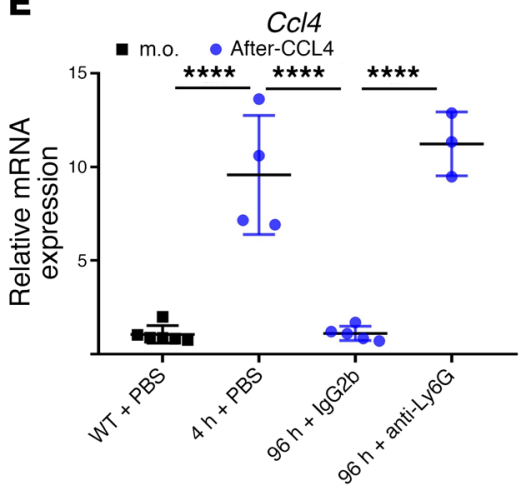

CD163
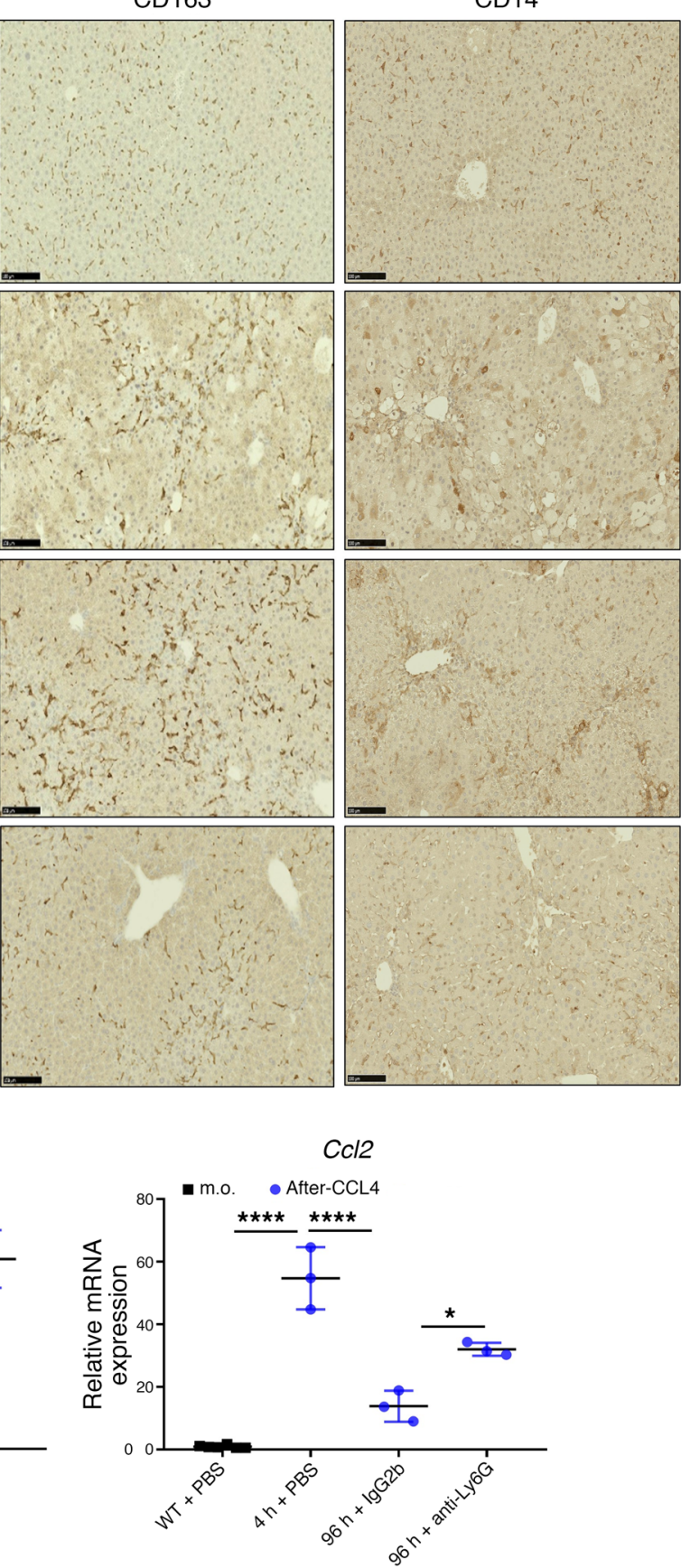

B

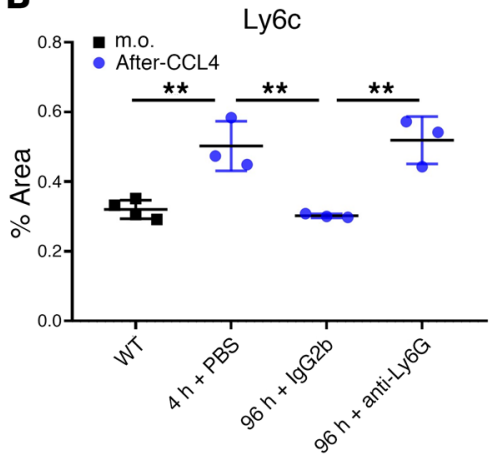

C

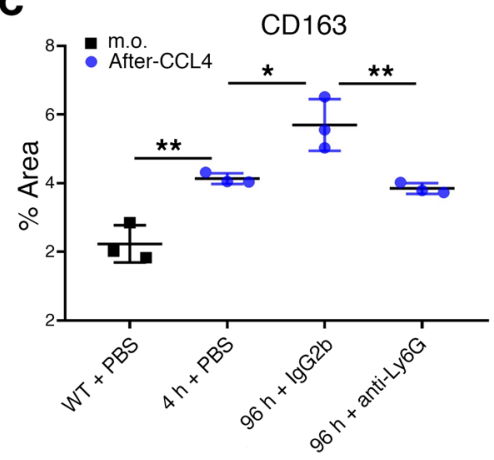

D

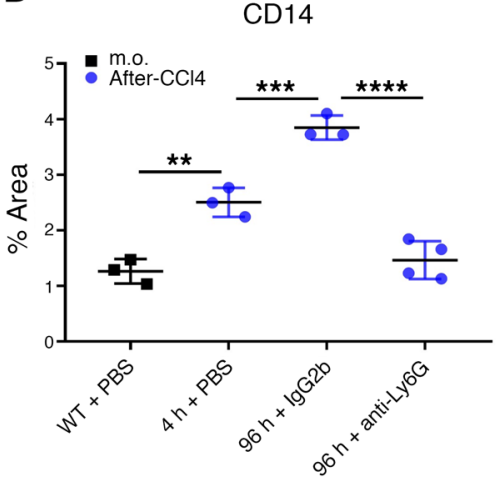

Figure 2. Neutrophil depletion results in persistence of classically activated hepatic macrophages during SRLI. (A) Representative microphotographs of dissected livers stained for proinflammatory, restorative, and antiinflammatory macrophages using antibodies against Ly6C, CD163, or CD14, respectively. Scale bars: $100 \mu \mathrm{m}$. (B-D) Area positive for Ly6C, CD163, or CD14 expressed as percentage and assessed with Image software in 10 aleatory selected images. ${ }^{*} P<0.05,{ }^{* *} P<0.01,{ }^{* *} P<0.001,{ }^{* * *} P<0.0001$, 1-way ANOVA, $n=3-4$ per group. (E and $\mathbf{F}$ ) Hepatic expression of $C c / 4$ and $C c / 2$ transcripts as measured by quantitative RT-PCR, relative to B2m mRNA housekeeping gene. ${ }^{*} P<0.05$, ${ }^{* * *} P<0.0001,1$-way ANOVA. Data are represented as means \pm SD, $n=3-6$ per group. Representative experiment of 2 independent repeats.

ulated, especially Mmp8, in mice given anti-Ly6G mAb compared with control mice that did not receive anti-Ly6G mAb (Figure 3 , D and E). By contrast, the transcript levels of the profibrotic gelatinase Mmp9 after treatment with anti-Ly6G mAb showed an increasing tendency compared with IgG2b isotype control (Figure 3F). We also found transcript levels of Timp1, a positive regulator of fibrogenesis, to be substantially augmented in anti-
Ly6G-treated mice compared with control mice untreated with anti-Ly6G mAb (Figure 3G).

Neutrophil abrogation impaired spontaneous resolution of inflammation and advanced fibrosis in NASH. To elucidate the role of neutrophils beyond spontaneous resolution of inflammation and fibrosis induced by toxin (CCL4), a dietary model of methionine and choline deficiency (MCD) that well resembles human 
fibrotic NASH was developed for 6 weeks, and anti-Ly6G mAbmediated neutrophil depletion (Supplemental Figure 8, A and B) was conducted during a 2 -week cessation period after the feeding course (spontaneous resolution period; Figure 4A). MCD-fed mice treated with IgG2b isotype and allowed to resolve spontaneously served as neutrophil depletion controls, while MCD-fed mice not allowed to resolve and given PBS represented the inflamed and fibrotic controls. Mice fed with methionine-supplemented diet (MCS) served as WT controls. After 2 weeks of cessation of the MCD feeding, depletion of neutrophils led to a significant decrease of hepatic accumulation of antiinflammatory macrophages (CD163 positive) as compared with IgG2b treatment control (Figure 4, B and E). This was accompanied by a modest diminution of the abundance of total macrophages ( $\mathrm{F} 4 / 80$ positive; Supplemental Figure 9A) and an increasing trend of the levels of Tnfa, Il12, and Nlrp3 transcripts (Supplemental Figure 9, B-D), indicating a worsened inflammatory scenario.

Notably, treatment with anti-Ly6G mAb during cessation of MCD dieting further impaired the hepatic architecture, as shown by a significant increase of the activation of HSCs ( $\alpha$ SMA positive) and a substantial accumulation of total collagen versus treatment with IgG2b (Figure 4, B-D), determining an impairment of fibrosis resolution.

Collectively, these results point toward a resolutive role of neutrophils during the cessation of injury dieting in NASH.

miR-223 loss of function worsens SRLI and early fibrosis. The granulocyte-specific microRNA miR-223 has recently emerged as an endogenous post-transcriptional silencer of the proinflammatory sensor NLRP3 through its 3 '-UTR $(18,20)$. To elucidate the underlying mechanism(s) by which neutrophils revert the activation of proinflammatory macrophages in the liver, miR-223 $3 p$ and Nlrp3 were both measured by real-time reverse transcriptase PCR (RT-PCR) in hepatic macrophages isolated from our experimental mice shown in Figure 1. Neutrophil abrogation led to a significant downregulation of hepatic macrophage miR-223 $3 p$ levels compared with the expression of miR-223 3p in hepatic macrophages of non-neutrophil-depleted mice (Figure 5A). Consistent with these data, hepatic macrophages from neutrophildepleted mice showed a very significant upregulation of Nlrp3 mRNA levels in hepatic macrophages versus miR-223 3p transcripts from the same mice (Figure $4 \mathrm{~A}$ ); this effect was not observed in the hepatic macrophages isolated from non-neutrophil-depleted mice (Figure 5A).

CD177 is a surface membrane coreceptor specifically expressed in granulocytes (35-38). It is principally involved in neutrophil migration (35), and it has been found to be carried in extracellular vesicles (EVs), particularly microvesicles, released by neutrophils (38).

Because microvesicles are increasingly recognized as endogenous carriers of microRNAs (39) and macrophages and neutrophils are within close vicinity in the hepatic sinusoids (40-43), we assessed the presence of CD177 in hepatic macrophages of WT, miR-223 ${ }^{--}$, and anti-Ly6G mAb-treated mice during SRLI by staining with an anti-CD177 antibody to verify whether a neutrophil-mediated delivery could be the source of the miR-223 increase we found in hepatic macrophages of non-neutrophildepleted mice during SRLI (Figure 5A). The total number of anti-
CD177-positive cells was higher in the hepatic macrophages from WT control mice than in those from anti-Ly6G mAb-treated or miR-223/- mice within the inflammation resolution period (Supplemental Figure 10, A and B, and Supplemental Figure 11A). Importantly, no fluorescent signal was found in anti-CD177stained or unstained hepatic macrophages from a separate group of untreated WT mice, confirming nonconstitutive expression of CD177 or autofluorescence (under 488 UV filter) in hepatic macrophages under basal conditions (Supplemental Figure 11, A and B). These results suggest the presence of CD177 in hepatic macrophages during the inflammation resolution period, and therefore point toward a potential miR-223 delivery to hepatic macrophages via neutrophilic EVs.

To evaluate the role of miR-223 as a potential mediator of SRLI, miR-223-deficient mice (representing the miR-223/-- group) or their age- and sex-matched littermates (corresponding to the WT control group) were given CCL4, via oral gavage, following the same regimen as in Figure 1A but shortened to 4 days. After the last treatment with CCL4, WT or miR-223--- mice were euthanized immediately after a 96-hour recovery phase from the inflammatory effect of CCL4 (Figure 5B). A separate group of miR-223-/- mice was post-transcriptionally replaced with an i.v. injection containing $1 \mathrm{mg} / \mathrm{kg}$ of the synthetic miR-223 3p, 24 and 72 hours after the last CCL 4 treatment; this group represented the miR-223 3p-treated miR-223-- mice (Figure 5B). All mice were euthanized 24 hours after the last injection (96 hours after CCL4, end of resolution phase) (Figure 5B). A significant increase in serum ALT levels of miR-223 $3^{--}$mice (vs. WT controls) suggested an apparent antiinflammatory function of miR-223 during the resolution phase (Figure 5C), which was further supported when the miR-223 3p-treated miR-223-/ group reverted ALT levels to within the normal range (vs. miR-223/- mice) (Figure 5C). Compared with untreated WT mice, histopathological examination of miR-223-mice and their age- and sex-matched littermates revealed massive leukocyte infiltration, hepatocyte ballooning, and fibril septae formation, more prominently in miR-223-- mice, 4 hours after CCL4 (Supplemental Figure 12) as assessed by the Ishak scoring system (23); this effect was absent and ameliorated in WT and miR-223-1mice, respectively, 96 hours after CCL4 administration (Figure 5, $\mathrm{D}$ and $\mathrm{E}$ ), which indicates favorable resolution of inflammation within a greater time frame. Hepatic necroinflammation was resolved to a lesser extent in miR-223/- mice (vs. WT) 96 hours after CCL4 (Figure 5, D and E), while the administration of miR2233 p modestly reverted this pathology (Figure 5, D and E). Consistently with these data, the area occupied by anti-F4/80 antibody in the liver of miR-223-/- mice was significantly augmented (vs. WT controls), while treatment with miR-223 $3 \mathrm{p}$ restored it (Figure 5, D and F). Confirming these data, the serum from miR$223^{-/-}$mice exhibited upregulation of IL-12 cytokine in comparison with WT controls (Figure 5G), while mice treated with miR-223 3p displayed a modest decrease of serum IL-12 (Figure 5G), indicating worsening systemic inflammation.

To further evaluate the antiinflammatory role of miR-223 in the macrophage compartment during SRLI, hepatic sections of mice from the experiment shown in Figure $5 \mathrm{~B}$ were stained with antibodies for Ly6C, CD163, or CD14. miR-223/- mice exhibited an augmented positive area for Ly6C (vs. WT controls), while 
A

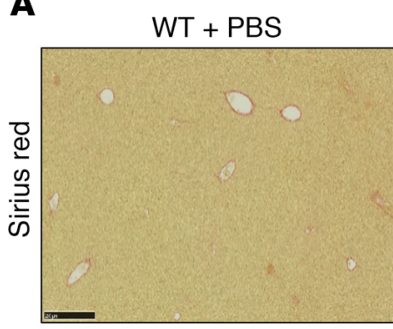

$4 \mathrm{~h}$ post-CCL 4 + PBS

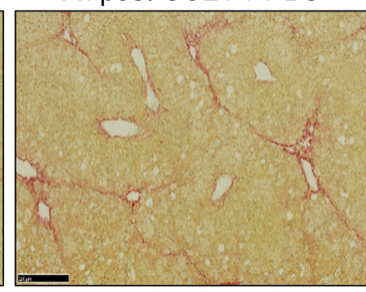

$96 \mathrm{~h}$ after-CCL $4+\lg$ G2b

$96 \mathrm{~h}$ after-CCL4 + anti-Ly6G

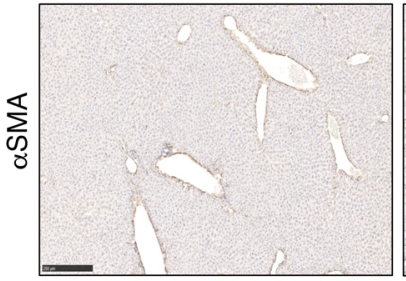

B

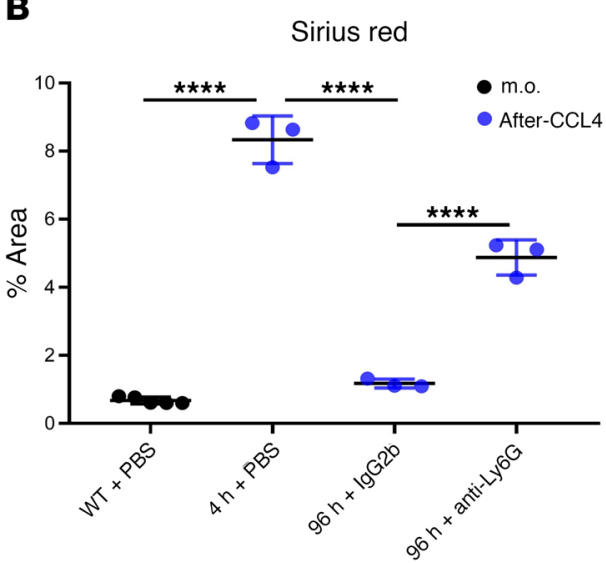

D

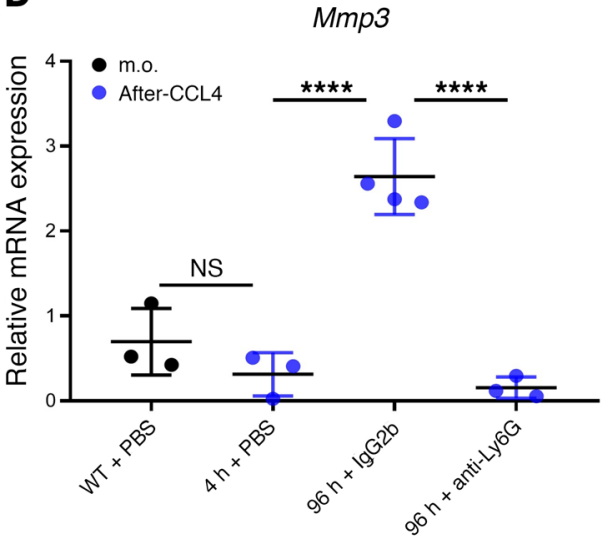

$\mathbf{F}$

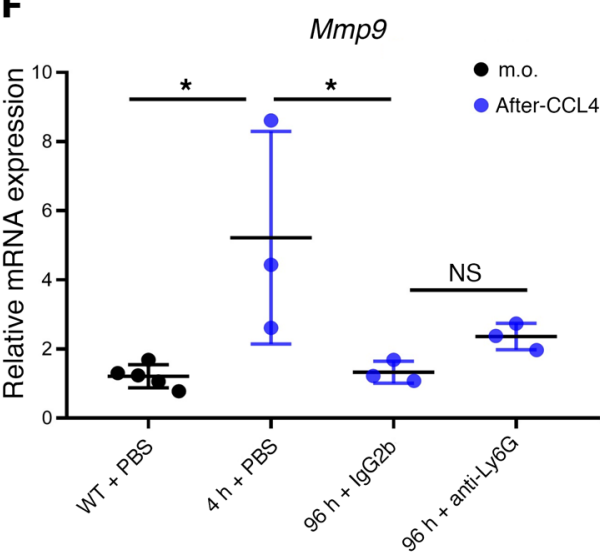

c

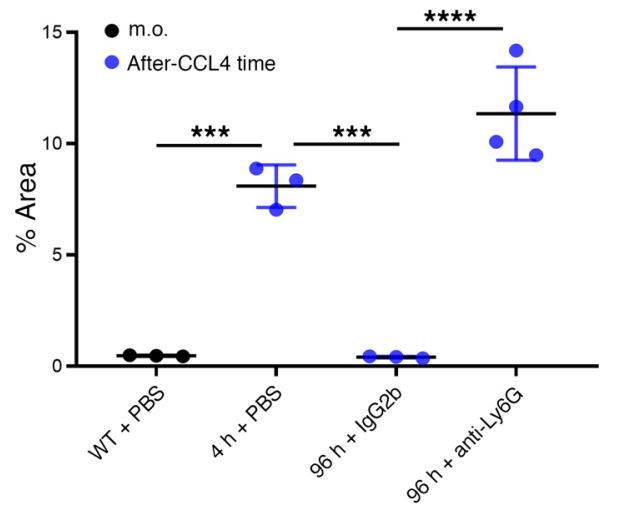

E

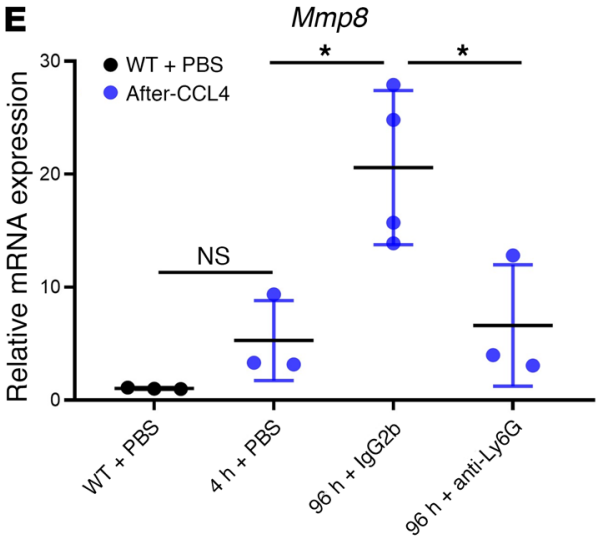

G

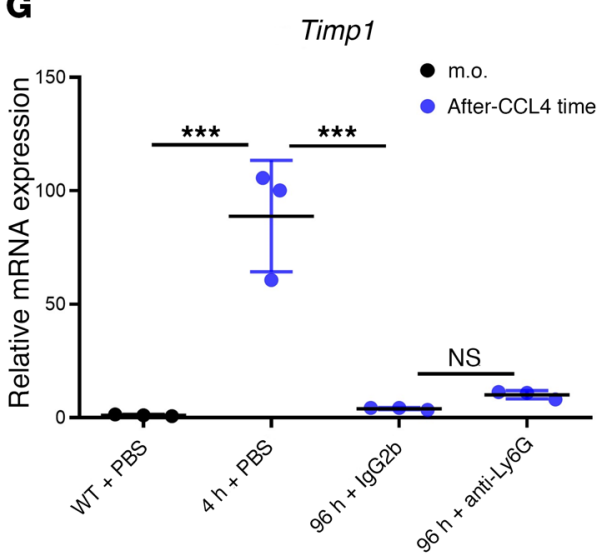

Figure 3. Neutrophil deletion aggravates spontaneous resolution of early liver fibrosis. (A) Sections from livers from the experiment in Figure $1 \mathrm{~A}$ displaying representative staining for collagen and for a subset of activated HSCs with Sirius red or anti- $\alpha$ SMA mAb, respectively. Scale bars: $250 \mu \mathrm{m}$. (B and C) Area of Sirius red and $\alpha$ SMA expressed as percentages of 10 aleatory selected microscopic fields, quantified by Image). ${ }^{* *} P<0.001,{ }^{* * *} P<0.0001$, 1-way ANOVA, $n=3-6$ per group. (D-C) Relative mRNA expression of $\mathrm{Mmp3}$, Mmp8, Mmp9, and Timp1 genes from liver homogenates of the experiment in Figure $1 \mathrm{~A}$, assessed by real-time RT-PCR and normalized to B2m mRNA housekeeping gene. ${ }^{*} P<0.05$, ${ }^{* *} P<$ $0.001,{ }^{* * *} P<0.0001,1$-way ANOVA, $n=3-6$ per group. Data are shown as means \pm SD. Representative experiment of 2 independent repeats. 
A
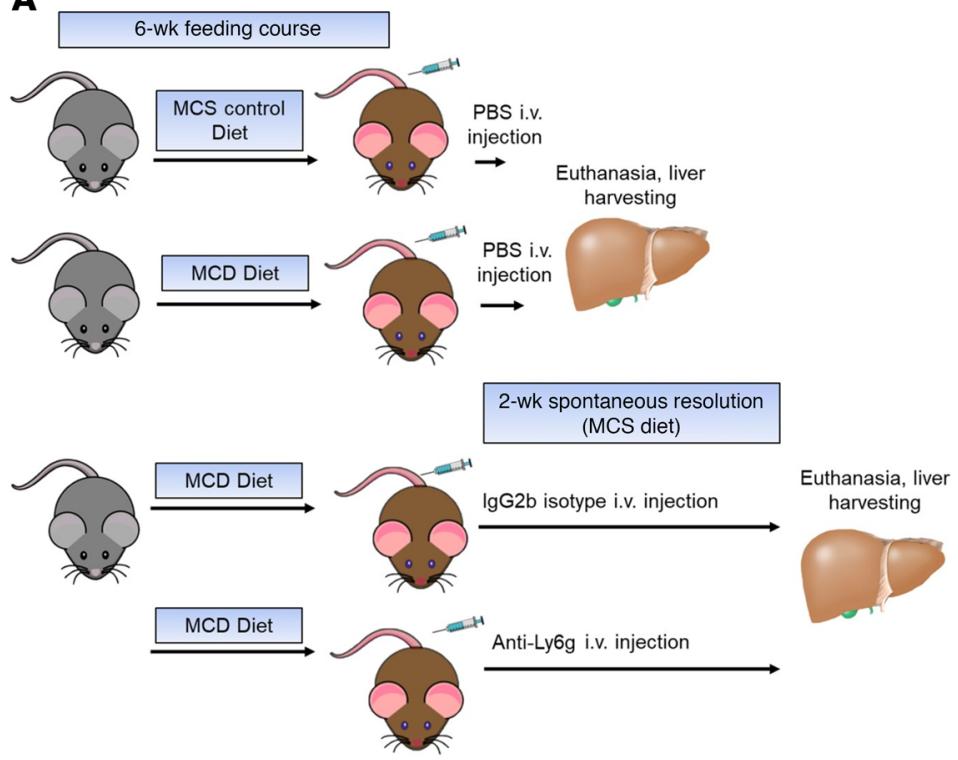

Figure 4. Neutrophil abrogation impairs the spontaneous resolution of inflammation and advanced fibrosis in NASH. (A) Experimental design showing neutrophil depletion with anti-Ly6G or control lgG2b mAb during 2 weeks (spontaneous resolution period) after a 6-week course of feeding with control MCS or MCD diet to induce fibrotic $\mathrm{NASH}$. Mice treated with PBS were euthanized immediately after feeding with MCD or MCS diet to serve as the inflamed or WT controls, respectively. (B) Representative IHC images of accumulated collagen, activated HSCs, and antiinflammatory macrophages stained with Sirius red or antibodies against $\alpha$ SMA or CD163, respectively. Scale bars: $100 \mu \mathrm{m}$. (C-E) Percentage of area positive for Sirius red, $\alpha \mathrm{SMA}$, or $\mathrm{CD163}$ calculated in 10 aleatory selected images. ${ }^{*} P<0.05,{ }^{*} P<0.01,1$-way ANOVA, $n=4-5$. Data are shown as means \pm SD. Representative experiment of 2 independent repeats.

B

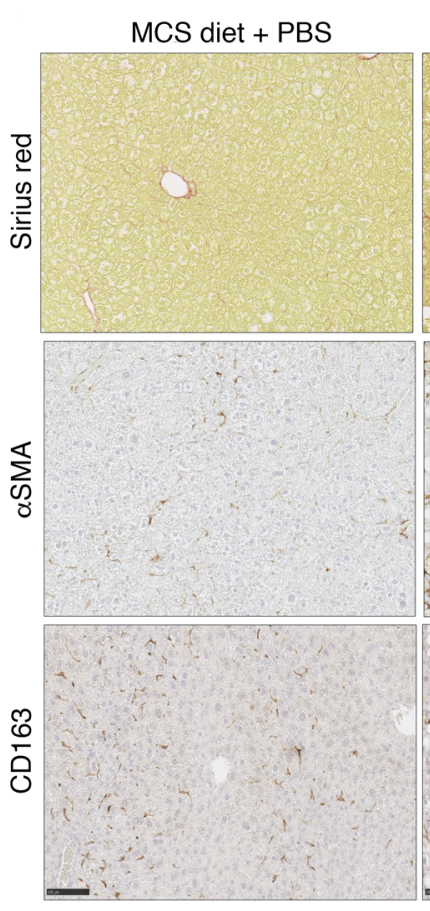

C

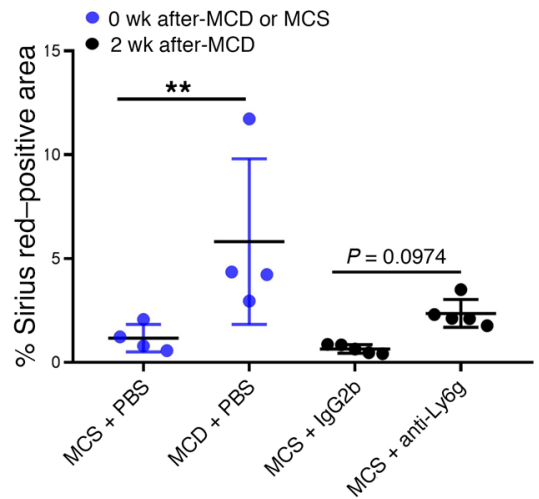

MCD diet + PBS
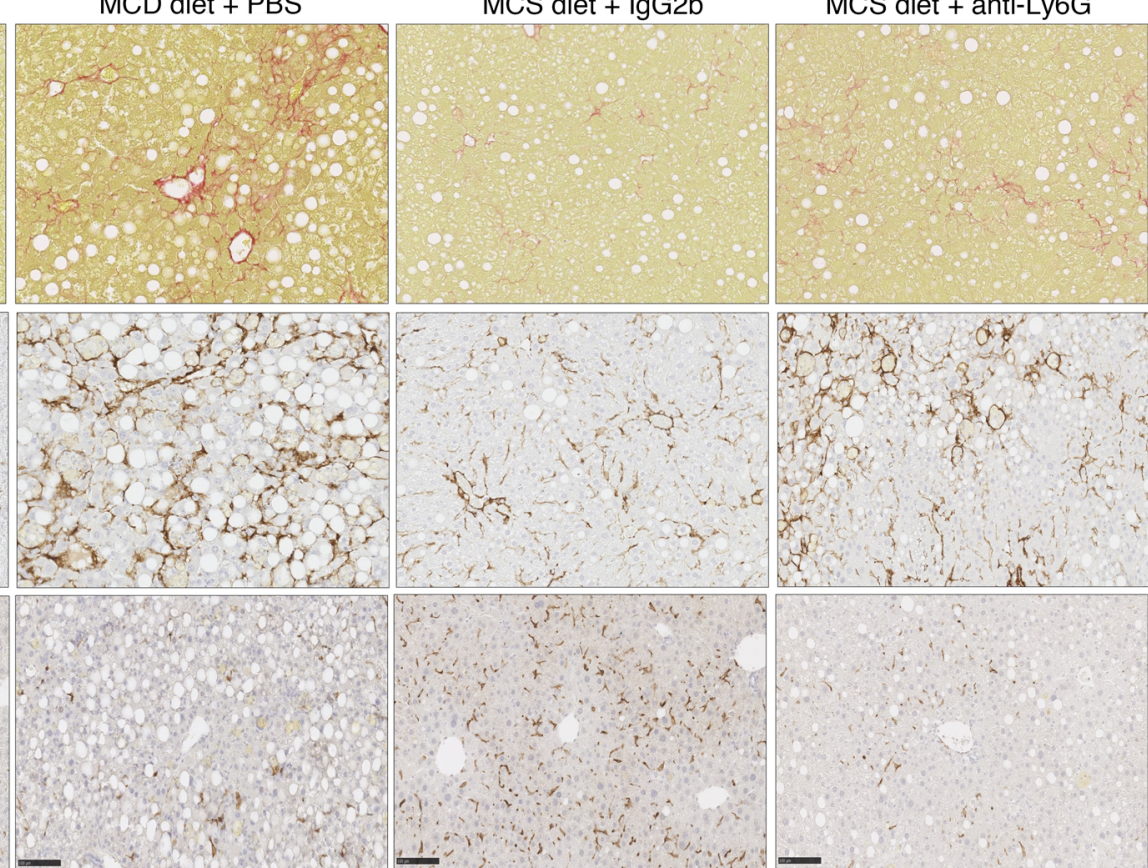

D

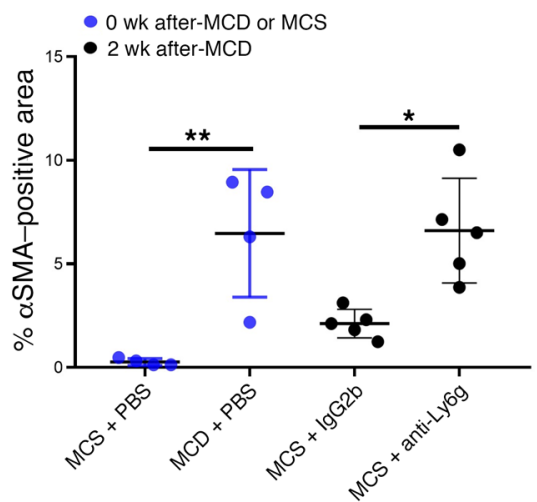

$\mathbf{E}$

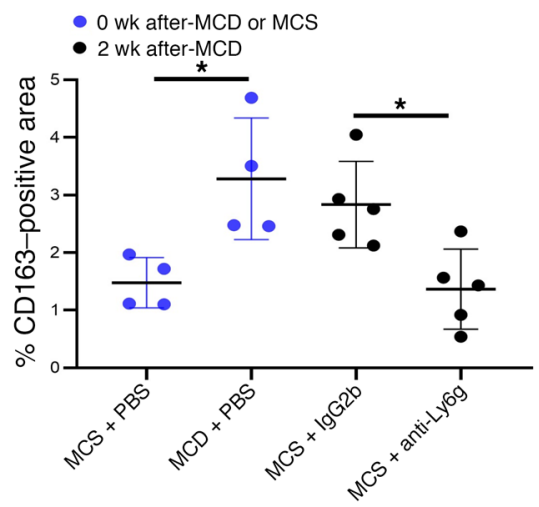


A

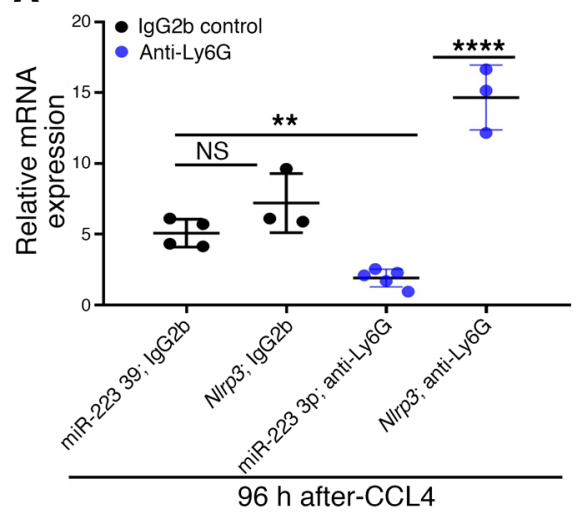

C

Serum ALT

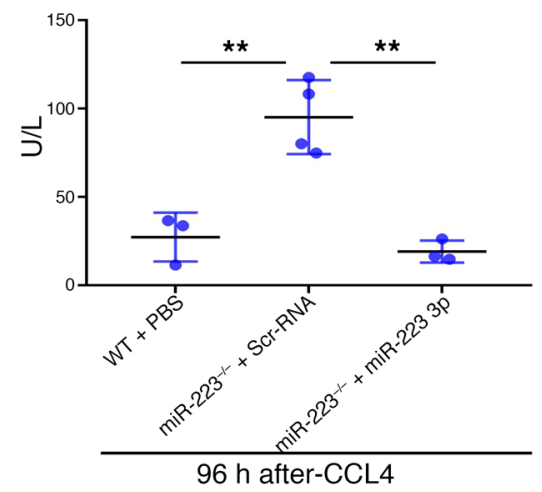

E

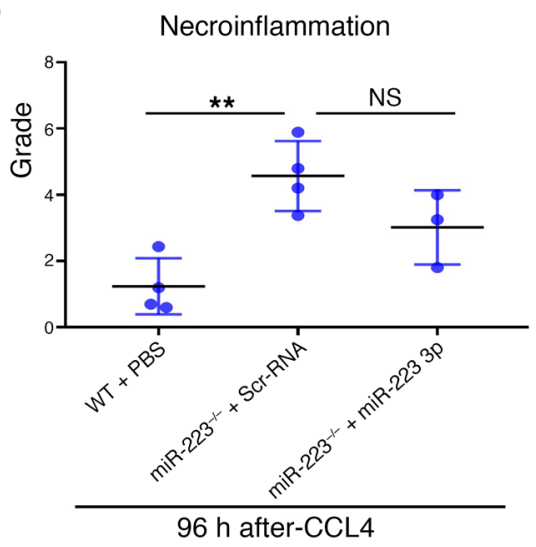

$\mathbf{F}$

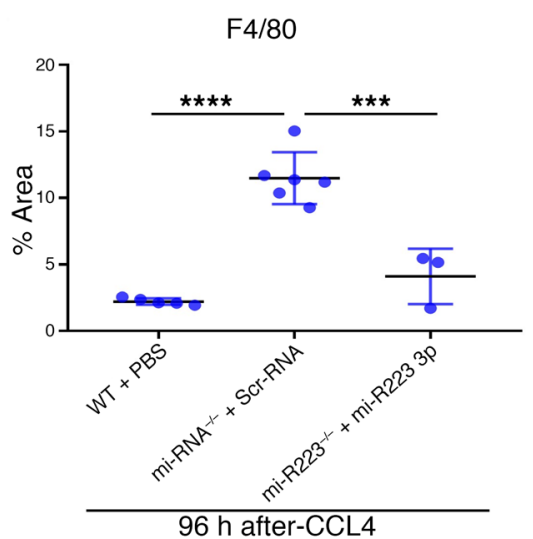

B

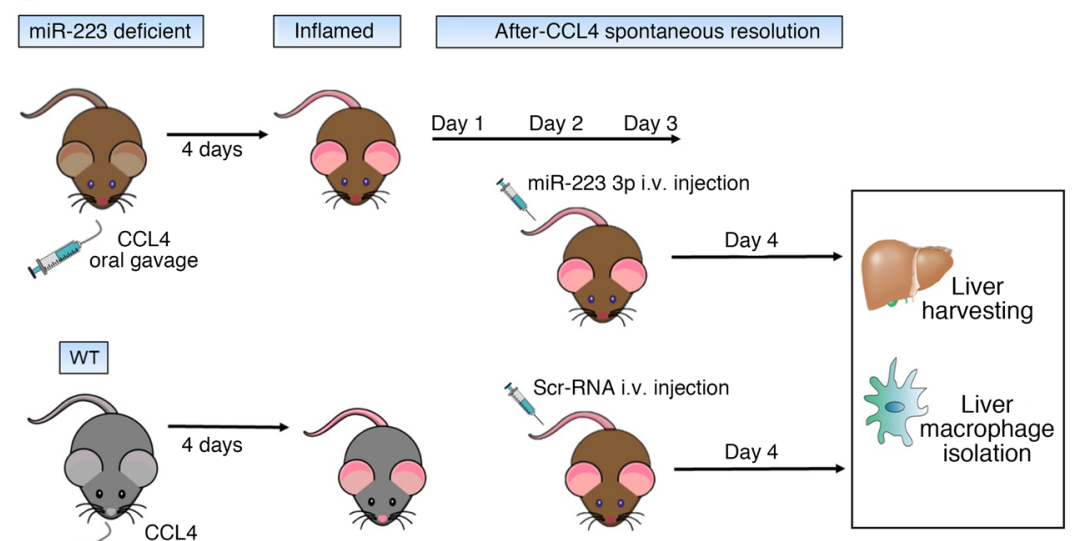

D

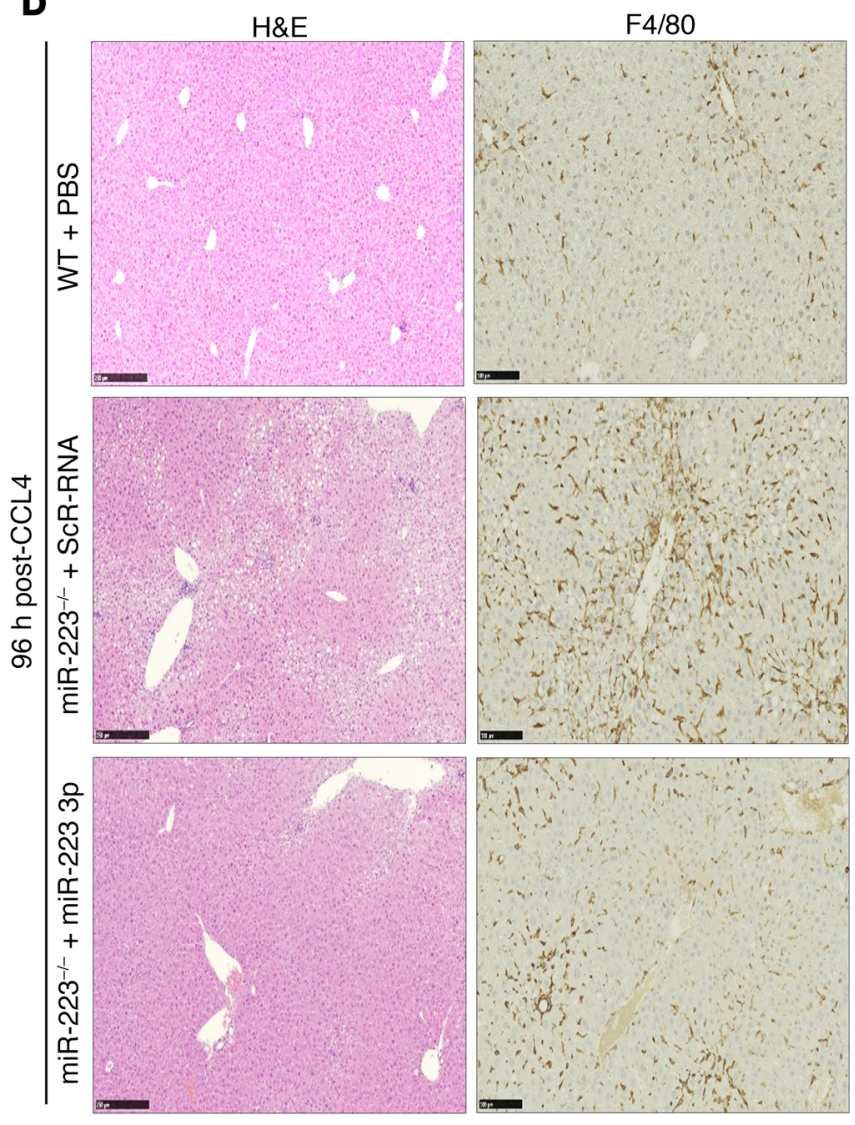

G

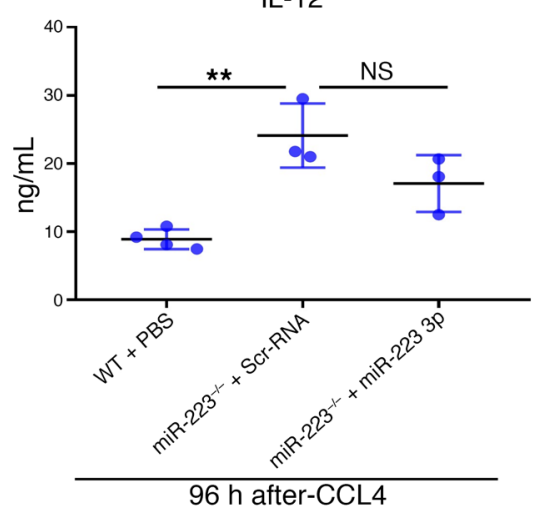


Figure 5. Regulation of SRLI by miR-223. (A) Expression of miR-223 3p and NIrp3 transcripts in isolated macrophages from livers of the experiment in Figure $1 \mathrm{~A}$ assessed by real-time RT-PCR and normalized to U6 or $B 2 m$ housekeeping genes, respectively. ${ }^{* *} P<0.01$, ${ }^{* * *} P<0.0001$, 2-way ANOVA, $n=3-5$ per group. (B) Experimental design of the miR-223 posttranscriptional replacement model during spontaneous recovery from liver inflammation after CCL4 treatment. (C) Levels of ALT in serum as measured by colorimetry. ${ }^{* *} P<0.01$, 1-way ANOVA, $n=3-4$ per group. (D) Liver sections representing images of liver cells and total macrophages after staining with $\mathrm{H} \& \mathrm{E}$ and anti-F4/80 antibody, respectively. Scale bars: 250 or $100 \mu \mathrm{m}$ for $\mathrm{H} \& \mathrm{E}$ or $\mathrm{F} 4 / 80$ images, respectively. (E) Necroinflammatory grade examined according to the Ishak system. ${ }^{*} P<0.01$, 1-way ANOVA, $n=3-4$ per group. (F) Percentage of area positive for $F 4 / 80$ in 10 randomly chosen images quantified by Image]. ${ }^{* *} P<0.001,{ }^{* * *} P<0.0001$, 1 -way ANOVA, $n=3-6$ per group. (C) Serum levels of IL-12 measured by colorimetric ELISA. ${ }^{* *} P<0.01,2$-tailed unpaired $t$ test, $n=3-4$ per group. Data are shown as means $\pm \mathrm{SD}$.

miR-223-/- treated with miR-223 3p overcame this effect (Figure 6, A and B) and displayed an expanded CD163-positive area (Figure 6, A and C). Similarly, the area stained for CD14 was markedly reduced in miR-223/- mice (vs. WT controls) and notably increased in miR-223 $3^{--}$mice treated with miR-223 3p (vs. miR$223^{-/}$) (Figure 6, A and D), further supporting the pivotal role of miR-223 during SRLI. Moreover, $\mathrm{Ccl} 2$ and $\mathrm{Ccl} 4$ mRNA expression levels were upregulated in miR-223-/- (vs. WT controls) and down-

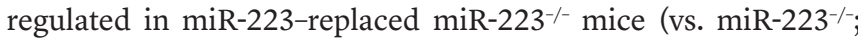
Figure 6, E and F), suggesting a de novo infiltration of proinflammatory macrophages/monocytes that is augmented when miR223 is absent in the liver and abrogated when miR-223 is posttranscriptionally replaced with miR-223 3p treatment.

Together, these results are in alignment with those found under neutropenia conditions during SRLI, suggesting that miR223 might play a pivotal role in neutrophil-associated resolution of inflammation after cessation of an exogenous stressor.

To further uncover the link between neutrophils and the spontaneous resolution of early liver fibrosis, total collagen and HSC activation were assessed in liver sections of WT control, miR-223--, or miR-223 3p-treated miR-223-- mice from the experiment shown in Figure 5B. The areas positive for Sirius red or anti- $\alpha \mathrm{SMA}$ within the liver parenchyma of miR-223 $3^{-/-}$mice were significantly expanded in comparison with WT control mice (Supplemental Figure 13, A-C). By contrast, the liver architecture of miR-223\%mice treated with miR-223 3p significantly improved in comparison with WT controls, as shown by an overall reduction in the area stained positive for Sirius red or anti- $\alpha$ SMA antibody (Supplemental Figure 13, A-C). Correlating these results, we found the expression of both $M m p 3$ and $M m p 8$ to be dramatically downregulated in $\mathrm{miR}-223^{-/-}$mice versus WT controls, and upregulated in miR$223^{-/-}$mice treated with miR-223 3p (vs. miR-223 $3^{-/-}$mice; Supplemental Figure 13, D and E); this indicates that collagen deposition is exacerbated by the absence of miR-223 during the spontaneous resolution phase of early liver fibrosis.

To confirm that endogenous, antiinflammatory miR-223 has a neutrophilic source in liver during SRLI, neutrophils isolated from the peritoneal cavity of WT (WT neutrophils) or male, untreated, miR-223-deficient mice (miR-223-/y Neu) were infused i.v. to female miR-223 ${ }^{-/-}$mice during resolution phase
(96 hours) after a 1-week course of CCL4 gavage (Figure 7A); CCL4-treated miR-223 $3^{-/-}$mice infused with miR-223-/y Neu represented the inflammation controls, while those infused with PBS served as the neutrophil infusion control group. One day later, CCL4-treated miR-223/- mice infused with WT neutrophils showed significantly decreased collagen deposition, modestly reduced populations of total macrophages ( $\mathrm{F} 4 / 80$ positive) and monocytes (Ly6C positive), and significantly increased accumulation of antiinflammatory macrophages (CD163 positive) in the liver parenchyma as compared with controls infused with untreated $\mathrm{miR}-223^{-/ y} \mathrm{Neu}$ (Figure 7, B-F).

Collectively, these results denote a novel contribution of neutrophils, via miR-223, in the spontaneous resolution of inflammation and liver injury after the causative insult has been ceased.

miR-223 deficiency hinders alternative activation of hepatic proinflammatory macrophages during SRLI. To uncover the cellular mechanism by which miR-223 participates in SRLI, total hepatic macrophages isolated from untreated miR-223 $3^{-/-}$mice or their WT controls were analyzed by FACS to determine the percentage of total, proinflammatory, and restorative macrophage populations (gating strategy shown in Supplemental Figure 14). The total hepatic macrophage population (CD11b-positive cells) of miR-223-/mice was predominantly composed of proinflammatory macrophages (double Ly6C- and F4/80-positive cells) (Figure 8, A and $\mathrm{B}$ ), while restorative, alternatively activated, macrophages (double CD206- and F4/80-positive cells) were the dominant population in WT controls (Figure 8, A and B). These data were confirmed by a significant expansion of the proinflammatory hepatic macrophage population (double Ly6C- and F4/80-positive cells) in miR-223-/mice compared with the same population in the WT control mice (Figure 8, A and B). The levels of iNos mRNA were upregulated in the hepatic macrophages of miR-223-/- mice, while Arg1 transcripts were notably decreased (Supplemental Figure 15). This effect was not observed in the hepatic macrophages taken from miR-223 3ptreated mice, as these mice displayed an inverse mRNA expression pattern where Arg1 was enhanced and iNos was decreased, when compared with miR-223-/- mice (Supplemental Figure 15).

These results indicate that miR-223 mediates alternative activation of hepatic proinflammatory macrophages during SRLI.

miR-223 deletion exacerbates NLRP3 expression in hepatic macrophages during SRLI. To further elucidate the molecular mechanism that miR-223 uses to induce a restoring phenotype in hepatic macrophages during SRLI, isolated hepatic macrophages from WT control, miR-223--, or miR-223 3p-treated miR-223/-- mice were subjected to gene expression analysis of miR-223 3p and Nlrp3 (Figure 9A). Hepatic macrophages of miR-223-/- mice exhibited notably higher expression of Nlrp3 mRNA versus WT controls, while those of miR-223-/- mice treated with miR-223 3p dramatically reduced this effect in comparison with miR-223-/- mice (Figure 9A). Confirming these data, Nlrp3 transcripts were abruptly augmented in hepatic macrophages of $\mathrm{miR}-223^{-/-}$mice relative to their own expression levels of miR-223 3p (Figure 9A). This effect was not observed in the hepatic macrophages from miR-223-/- mice supplemented with miR-223 3p, which displayed a very significant downregulation of Nlrp3 mRNA versus their miR-223 3p expression levels (Figure 9A). To further verify these results, we used confocal microscopy to assess the expression pattern of NLRP3. The per- 
centage of cells positive for NLRP3 was significantly increased in miR-223 $3^{-1-}$ mice compared with the WT controls (Figure 9, B and C; merges shown in Supplemental Figure 16). Conversely, the miR-223 $3 \mathrm{p}$-treated miR-223/- mice exhibited a diminution of NLRP3-positive cells versus miR-223/- mice (Figure 9, B and C; merges shown in Supplemental Figure 16). These results further prove that miR223 regulates NLRP3 post-transcriptionally during SRLI.

Functions beyond inflammation have been attributed to the p50 subunit of the transcription factor NF- $\kappa B$ owing to its regulation of IL-10 transcription $(44,45)$. To explore the upstream effect that miR-223-induced Nlrp3 silencing might exert on hepatic macrophages during SRLI, the mRNA expression of $N f k b$ p50 and IllO was measured in hepatic macrophages from WT, miR-223--, and miR-223 3p-treated miR-223 $3^{-/}$mice. The levels of $N f k b$ p50 and Il1O mRNA were robustly diminished in miR-223 $3^{--}$mice compared with their respective WT control mice (Figure 9, D and $\mathrm{E})$. In contrast, the levels of $\mathrm{N} f k b \mathrm{p} 50$ and $\mathrm{Il1O}$ transcripts in miR$223^{-/-}$mice treated with miR-223 $3 \mathrm{p}$ were substantially increased versus miR-223 $3^{--}$mice (Figure 9, D and E); these results suggest that the alternative activation of hepatic macrophages during SRLI might involve miR-223-mediated silencing of NLRP3 that in turns upregulates $N f k b$ p50 and subsequently Il1O.

Conclusively, during SRLI, our data collectively exhibit neutrophilderived miR-223 as a silencer of Nlrp3 in hepatic macrophages, which in turn polarize to a restorative phenotype that reduces the release of IL-10, thus mitigating fibrogenesis by impairing the activation of HSCs and collagen synthesis (Figure 10).

\section{Discussion}

Inflammation is a physiological response to tissue injury or infection that leads to the secretion of various inflammatory mediators, such as cytokines, chemokines, and eicosanoids, that are responsible for coordinating the mechanisms of cellular defense and tissue repair (46). A persistent inflammatory state results in chronic adaptations that in turn exacerbate tissue injury and may result in an abnormal wound healing response contributing to the development of liver fibrosis (47). The natural, spontaneous resolution of inflammation is now recognized as a highly coordinated cellular event characterized by a phenotypic transition of proinflammatory macrophages into restorative macrophages under the control of endogenous pro-resolving mediators that enable the restoration of tissue homeostasis (48). Information regarding the molecular events that mediate the resolution of ongoing inflammation in the liver is limited to specialized pro-resolving mediators, a novel class of autacoids that act as "stop signals" of the inflammatory response, including molecules such as n-3-derived resolvins, protectins, and maresins, as well as arachidonic acid-derived (n-6) lipoxins (49-51). Defective pro-resolving mechanisms may represent important novel contributors to persistent hepatic inflammation and suggest the possibility that abrogation of tissue inflammation could be achieved by exploitation of active naturally occurring pro-resolving mechanisms instead of the classical passive blockade of proinflammatory mediators.

While neutrophils have been canonically viewed as the first wave of defense against infection and tissue damage (52), they have been increasingly recognized as novel resolving cells during the wound healing response in various tissues, including the heart, skin, and joints (13-17). In the liver, neutrophil infiltration is a key histopathological feature of chronic liver conditions such as NASH and alcoholic liver disease $(53,54)$.

In addition, the extent of neutrophil infiltration in the liver has been recently recognized as a key positive prognostic factor in patients with alcoholic liver disease (55). However, the role of neutrophils during the resolution phase of inflammation, and the underlying mechanisms of that process, have yet to be explored. In our current study, our findings uncover a novel regulatory role of neutrophils as contributors to the spontaneous resolution of liver inflammation (SRLI) and identify miR-223 as a potential key mediator of these effects.

Using a well-established murine model of SRLI, we found that systemic neutrophil depletion in these mice leads to a worsening in overall liver inflammation and impaired transition of hepatic proinflammatory macrophages into a restorative phenotype both in vivo and ex vivo, and results in changes to the chronic inflammatory and advanced fibrosis stage of an MCD dietary model resembling human NASH. These findings led us to anticipate that a granulocyte-specific component must orchestrate the resolutive role that neutrophils acquire during SRLI.

miR-223 is a granulocyte-specific, short, noncoding, doublestranded RNA $(18,19)$ that has been identified as a post-transcriptional regulator of NLRP3 inflammasome activity $(18,20)$ - a critical danger sensor protein that is activated during tissue injury and mounts a robust monocyte/macrophage-driven inflammatory response via maturation and secretion of the proinflammatory cytokines IL-1 $\beta$ and IL-18 (56). Interestingly, miR223-knockout mice are highly sensitive to liver inflammation and injury when exposed to low-dose endotoxin injection (19). Moreover, untargeted hepatic microRNA profiling in patients with NASH has identified miR-223 as one of the top significantly downregulated microRNAs (21) in comparison with patients with fatty liver or normal healthy control patients, and proinflammatory macrophages from adipose tissue have been shown to polarize to a restorative phenotype through the action of miR-223 in obesityassociated chronic inflammation (57). Our results demonstrate that the suppression of miR-223 expression recapitulated the effects on inflammation resolution that we found via the depletion of neutrophils, and denoted a negative regulatory role of miR-223 in Nlrp3 mRNA expression in proinflammatory hepatic macrophages, thus suggesting the mechanism by which miR-223 mediates their alternative activation during SRLI. These data are consistent with previous published studies on extrahepatic macrophages under resolutive conditions (13-17).

miR-223, like any other RNA, may be present within the extracellular compartment in 2 distinct forms: either encapsulated in EVs, or in a non-membrane-bound form associated with specific proteins such as argonaute 2 (Ago2) and lipoproteins, which shield microRNAs from degradation (39). While naked or protein-bound microRNA cannot integrate into target cells because of its overall size and negative charges (39), EVs enable it to penetrate recipient target cells, acting as a safe and efficient delivery vehicle (39). Recent evidence has shown that neutrophil-derived EVs contribute to inflammation resolution in models of joint inflammation driven by NLRP3 inflammasome activation, in part by suppressing the response to Toll-like receptor (TLR) ligands in monocyte- 
A

$96 \mathrm{~h}$ after-CCL4
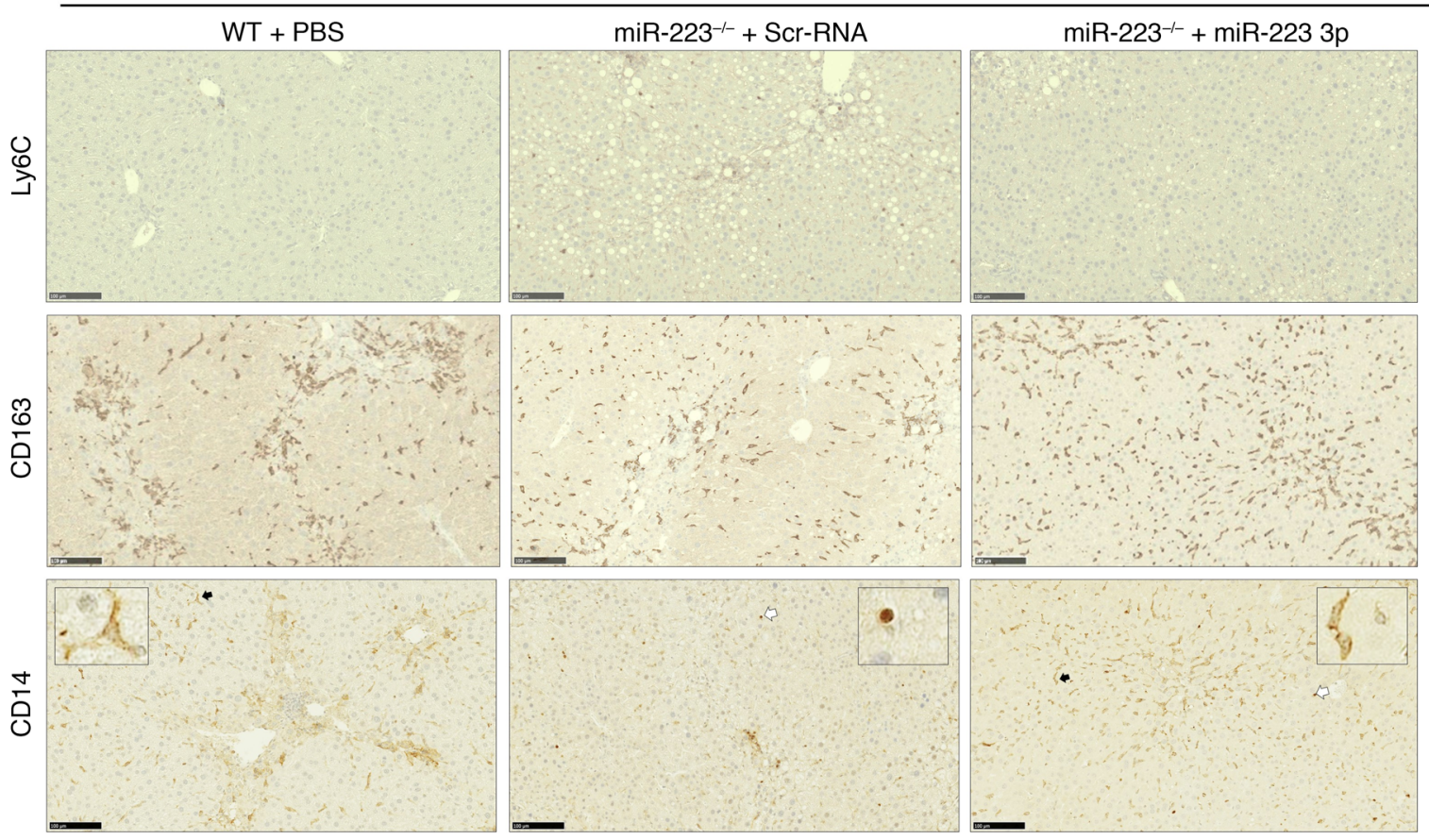

B

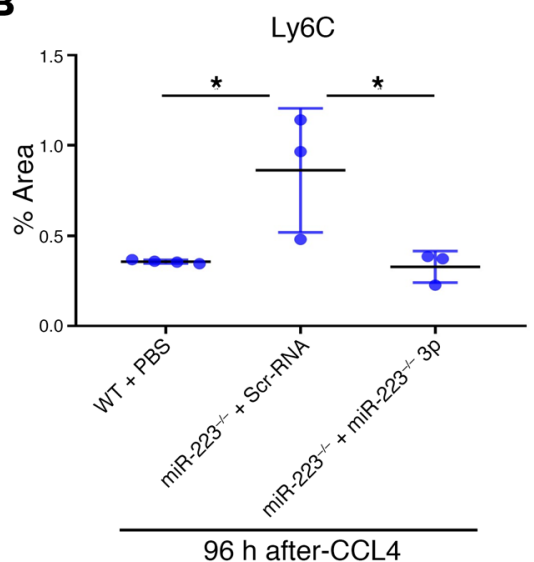

E

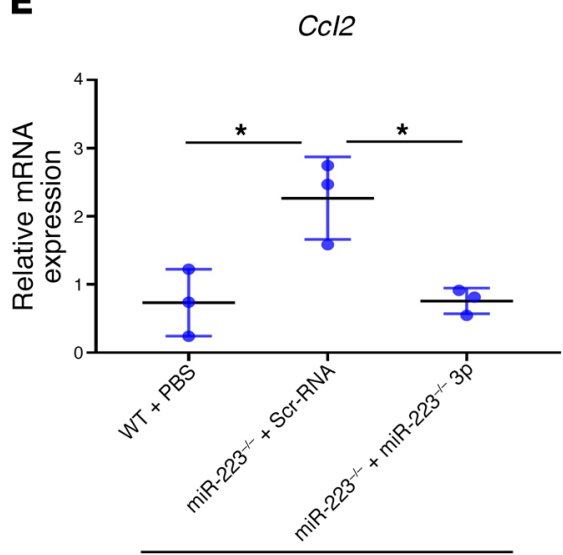

$96 \mathrm{~h}$ after-CCL4
C

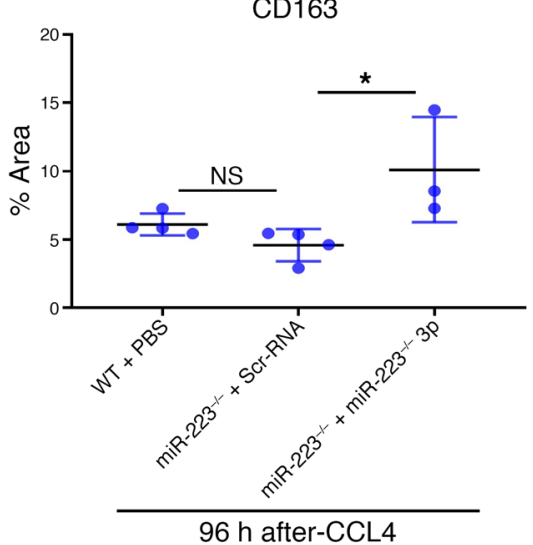

$\mathbf{F}$

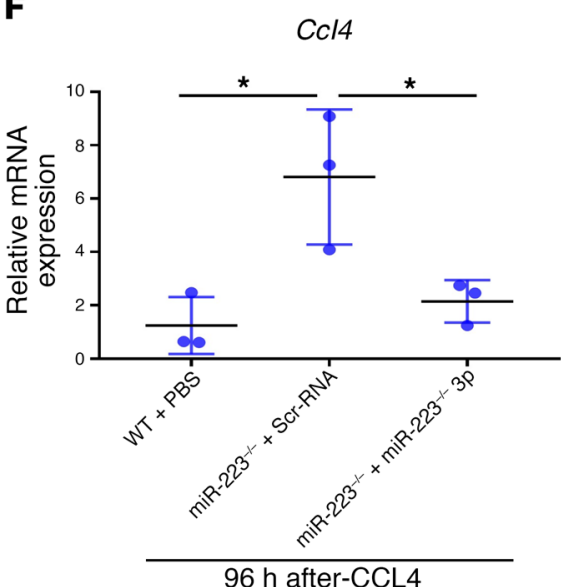

D

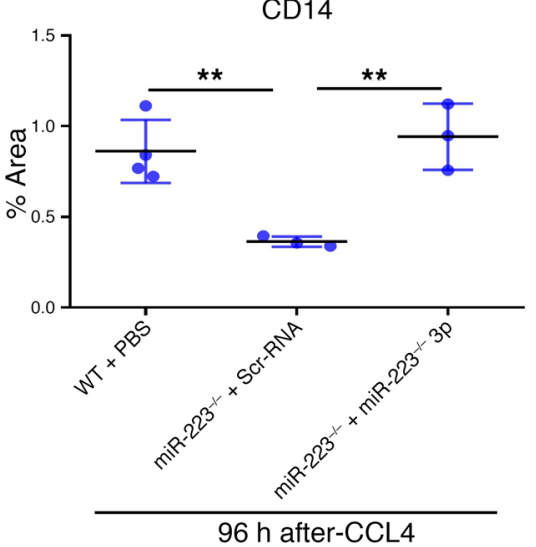

Figure 6. miR-223 deficiency reduces the hepatic restorative macrophage compartment in SRLI. (A) Representative pictures of liver sections exhibiting proinflammatory, restorative, and antiinflammatory macrophages as stained with anti-Ly6C, CD163, or CD14, respectively. Scale bars: $100 \mu \mathrm{m}$. (B-D) Percentage of area positive for Ly6C, CD163, and CD14 in 10 randomly chosen images quantified by Image). ${ }^{*} P<0.05,{ }^{* *} P<0.01,1$-way ANOVA, $n=3-4$ per group. (E and $\mathbf{F}$ ) $C \mathrm{Cl} 2$ and $\mathrm{Ccl} / 4$ mRNA expression normalized to $B 2 m$ mRNA and measured by real-time RT-PCR. ${ }^{*} P<0.05$, 1 -way ANOVA, $n=3$ per group. Data are shown as means $\pm \mathrm{SD}$. 
A

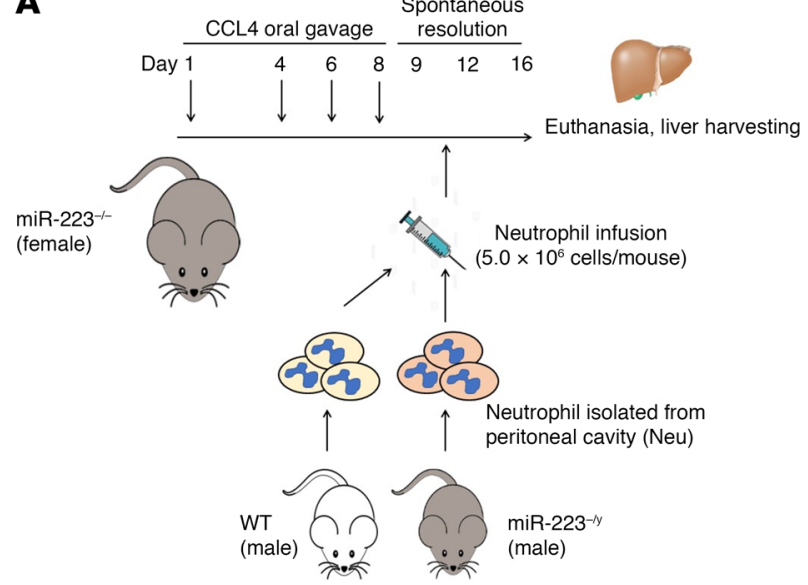

Figure 7. Neutrophils accelerate the spontaneous resolution of inflammation and fibrosis in $\mathrm{miR}$ 223-deficient mice. (A) Experimental design of WT or miR-223/- neutrophil infusion to miR-223/- mice during SRLI after a 1-week course of CCL4 gavage. (B) Representative micrographs of collagen accumulation, total macrophages, and monocytes stained with Sirius red or F4/80, Ly6C, or CD163 antibody, respectively. Scale bars: $250 \mu \mathrm{m}$. (C-F) Percentage of area positive for Sirius red, F4/80, Ly6C, or CD163 analyzed in 10 independent images. ${ }^{*} P<0.05,2$-way ANOVA, $n=3$. Results are displayed as means $\pm \mathrm{SD}$. Representative experiment of 2 independent repeats.

B

Spontaneous resolution after-CCL4

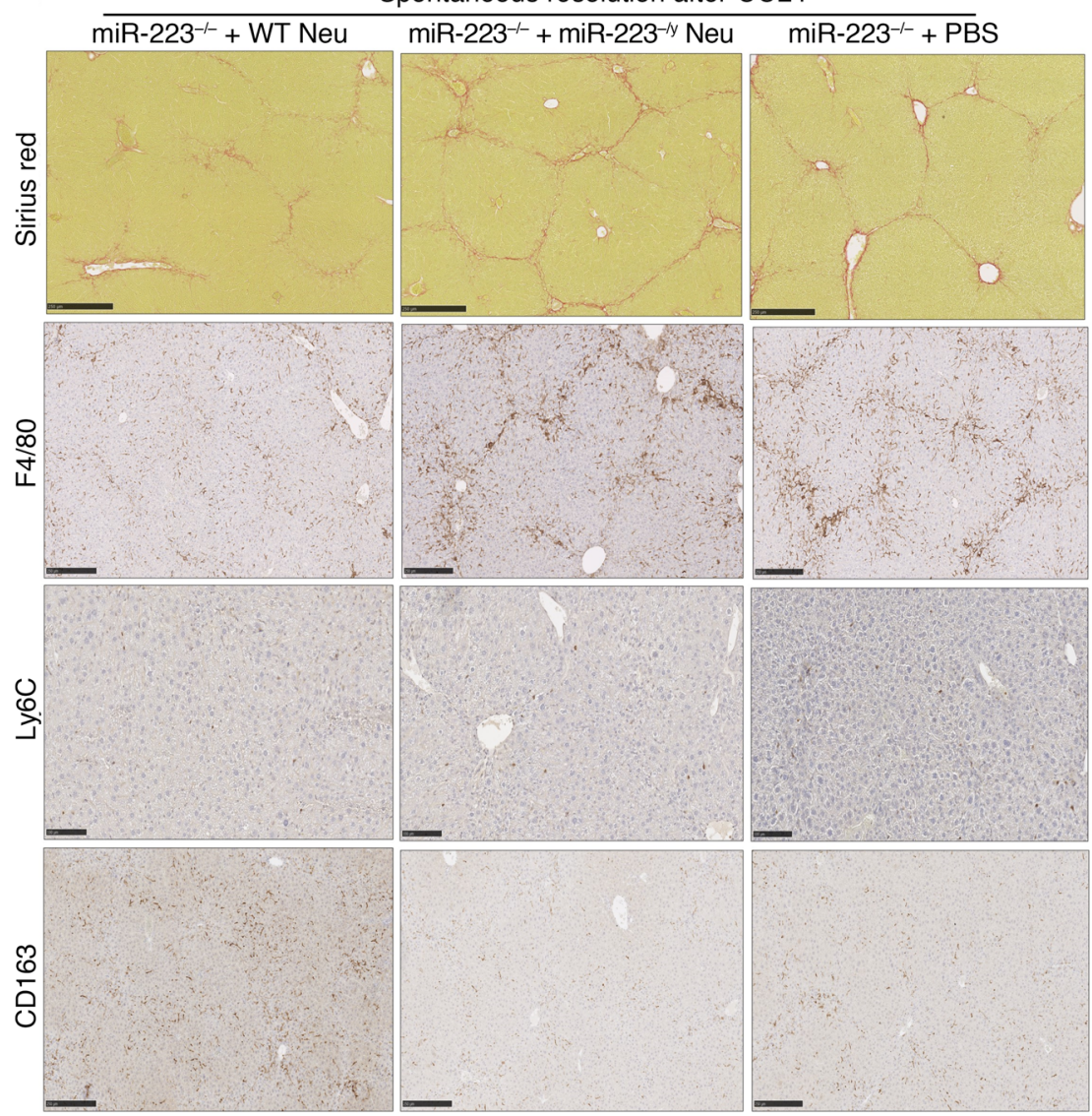

C

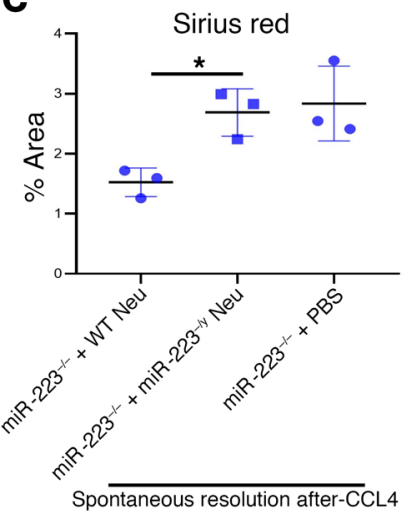

D

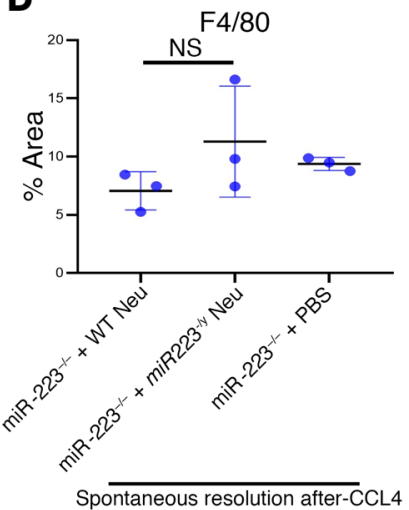

E

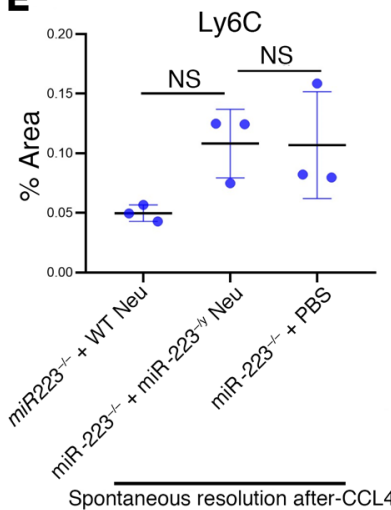

$\mathbf{F}$

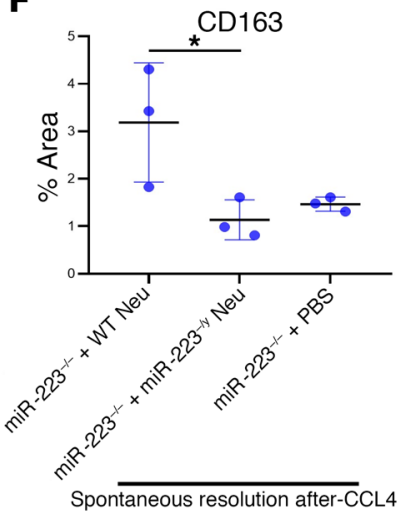


derived macrophages and dendritic cells $(15,16)$. Our data suggest that the transfer of miR-223 via EVs might represent a novel cellto-cell communication platform between neutrophils and macrophages. The close proximity of macrophages and neutrophils within the sinusoidal space of the injured liver parenchyma possibly facilitates this interaction (refs. 40-43 and Supplemental Figure 17). CD177 is a granulocyte-specific cell surface coreceptor that has been used as a specific marker of neutrophil-derived EVs (38). We observed the presence of CD177 in isolated hepatic macrophages of non-neutrophil-depleted mice during SRLI, while CD177 was absent in hepatic macrophages of both $\mathrm{miR}-223^{-/-}$and neutrophil-depleted mice during SRLI. These findings were associated with a reduced restorative hepatic macrophage compartment and an increase in the proinflammatory macrophage population in $\mathrm{miR}-223^{-/-}$mice, as well as the neutrophil-depleted mice, and a reduced inflammation and fibrosis after infusion of neutrophils isolated from WT mice to those undergoing SRLI in a miR-223-/- background, suggesting that neutrophils might induce a restorative phenotype in liver macrophages by delivering miR-223 within EVs.

The robust diminution in transcript expression levels of the antiinflammatory cytokine $I l 1 O$, and its transcription factor $N f k b$ p50, in hepatic macrophages of $\mathrm{miR}-223^{-/-}$mice carries the potential to be the upstream mechanism by which miR-223-derived Nlrp3 silencing possibly leads to an alternative activation of proinflammatory hepatic macrophages during SRLI without inducing changes in IL-1 $\beta$ expression (data not shown). Importantly, serum levels of IL-10 and the proinflammatory IL-12 cytokine were down- and upregulated, respectively, in mice that underwent systemic neutrophil depletion. This suggests an extrahepatic effect of neutrophils in the resolution of inflammation, which is consistent with previously published data (13-17).

The persistence of proinflammatory hepatic macrophages and liver inflammation that our data revealed in neutrophil-depleted, as well as miR-223-/, mice culminated in changes to early fibrosis patterns with significant fibrogenic activity and increased collagen deposition in the livers of these mice, thus supporting the link between persistent inflammation and liver fibrosis development. Treatment of miR-223/- mice with nanoparticle-delivered miR-223 $3 p$ restored inflammation resolution and protected against fibrosis. These results have significant translational implications for a variety of human hepatic, and potentially extrahepatic, disorders in which NLRP3-mediated persistent inflammation may represent an important driver of injury and fibrosis development.

Our data, together with previous evidence associating hepatic neutrophil infiltration with a positive outcome of alcoholic hepatitis (55), point toward a distinct phenotype of neutrophils directly influenced by the stage of the inflammatory response: classic, proinflammatory neutrophils remain as key players of acute response but might acquire a pro-resolving phenotype when the injury trigger ceases or when it persists but tolerance arises.

In conclusion, our findings uncover a role for neutrophils during the resolution phase of liver inflammation and identify miR223 as a novel, potential antiinflammatory and antifibrotic target.

\section{Methods}

Mice. Mouse studies were performed under permission and guidelines of the Institutional Animal Care and Use Committee (IACUC) of UCSD. Eight-week-old female C57BL/6N and male or female B6 Cd45.1 mice at 7-8 weeks of age were purchased from The Jackson Laboratory. Eight-week-old female or male miR-223 $3^{-/}$mice purchased from The Jackson Laboratory in a B6 Cd45.1 background were allowed to breed at $25^{\circ} \mathrm{C}$ housing with a 12-hour light/12-hour dark cycle; the allelic composition of these mice was Mir223tm1Fcam, and their background involved 129S4/SvJae and C57BL/6 mice.

SRLI after CCL4 intoxication. To induce liver inflammation, CCL4 diluted in mineral oil (m.o.) was given once to 8-week-old female C57BL $/ 6$ mice by oral gavage at $0.875 \mathrm{~mL} / \mathrm{kg}$. Three days later, the CCL4 dose was increased to $1.75 \mathrm{~mL} / \mathrm{kg}$ and was given every 3 days up to 1 week. Mice gavaged with m.o. served as noninflamed WT controls. A group of mice was euthanized 4 hours after CCL4 gavage to serve as inflamed controls, and 2 other groups were allowed to recover from inflammation for 4 days and euthanized immediately after.

SRLI in 7- to 8-week-old female or male miR-223-/- mice and their age- and sex-matched littermate controls (in a B6 Cd45.1 background) was induced as above but with the CCL4 regimen shortened from 9 to 4 days to avoid death particularly observed in these knockout mice.

Systemic neutrophil depletion in SRLI. To deplete neutrophils systemically during SRLI, the above C57BL/6 mice that were allowed to recover from inflammation after CCL 4 treatment were given 1 i.v. injection of PBS or monoclonal anti-Ly6G antibody (clone RB6-8C5; catalog 14-5931-82, Thermo Fisher Scientific) at $0.1 \mathrm{mg} / \mathrm{mouse}$ from day 1 to day 3 of the recovery period. One day later, the mice were euthanized. Mice injected with PBS served as inflammation recovery controls, while mice injected with rat IgG2b isotype control (catalog 40064, BioLegend) served as negative controls of neutrophil depletion.

Neutrophil depletion in the SRLI post-CCL4 model replicated for the revision of this paper was performed with clone $1 \mathrm{~A} 8$ of the antiLy6G mAb (catalog 127649, BioLegend).

SRLI after MCD dieting. Eight-week-old male C57BL/6N mice were fed ad libitum for 1 week, and, immediately after, the feeding was changed to a methionine- and choline-deficient (MCD) diet (A02082002B, Research Diets Inc.) or a methionine- and choline-supplemented (MCS) control diet (A02082003B, Research Diets Inc.) to induce fibrotic NASH. Six weeks later, mice were immediately euthanized to serve as MCD positive or MCS negative inflammation controls, and 2 independent groups fed with MCD were dieted with control MCS for 2 weeks to allow SRLI. Then, anti-Ly6G mAb (clone RB6-8C5, Thermo Fisher Scientific) or rat IgG2b isotype (BioLegend) was given i.p. at $200 \mu \mathrm{g} / \mathrm{mouse}$ every 4 days during the 2-week SRLI period; mice injected with IgG2b isotype represented the neutrophil depletion control. Twenty-four hours after the last injection, mice were sacrificed to harvest the liver, collect blood, and assess inflammation and fibrosis in liver biopsies.

miR-223 post-transcriptional replacement. An independent group of miR-223 $-1-$ mice treated with CCL4 and allowed to spontaneously recover from inflammation for 96 hours as described above were injected i.v. with miR-223 3p at $1 \mathrm{mg} / \mathrm{kg} 24$ hours and 72 hours after the last CCL4 treatment. A mimic of miR-223 was encapsulated as in vivo-ready mirVana mmu-miR-223 3p (mature sequence UGUCAGUUUGUCAAAUACCCCA) in Invivofectamine 3 (Thermo Fisher Scientific) according to the manufacturer's instructions, to allow safe systemic delivery.

Neutrophil isolation and infusion. Neutrophils were isolated from male $\mathrm{C} 57 \mathrm{BL} / 6 \mathrm{~N}$ mice or male miR-223-/y mice using a neutrophil (mouse) isolation kit (Cayman Chemical) according to the manu- 
A

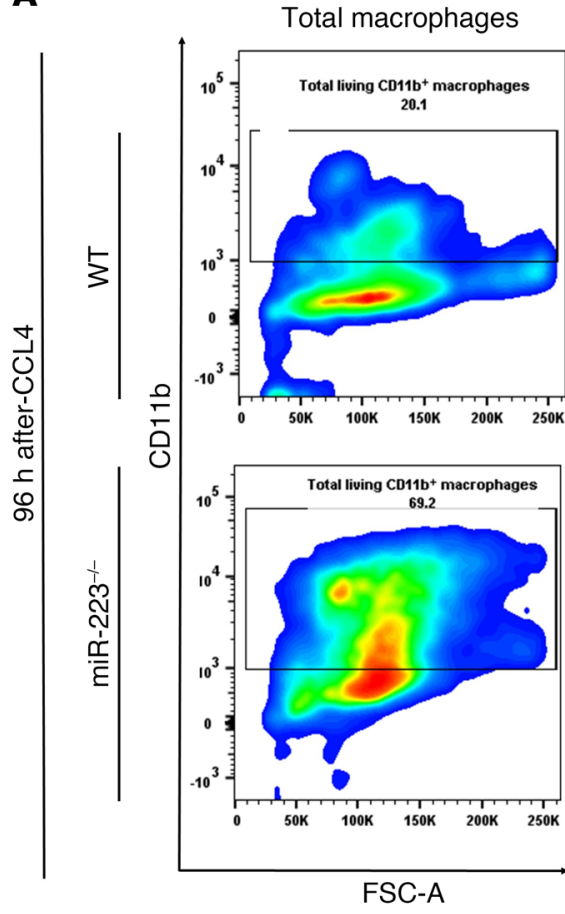

B

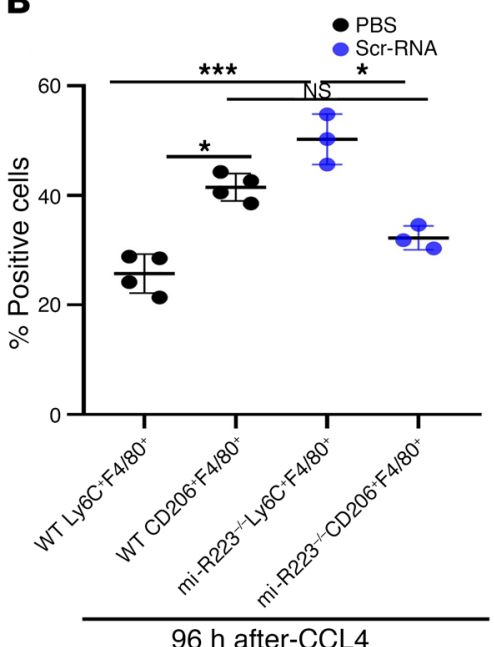

Proinflammatory macrophages
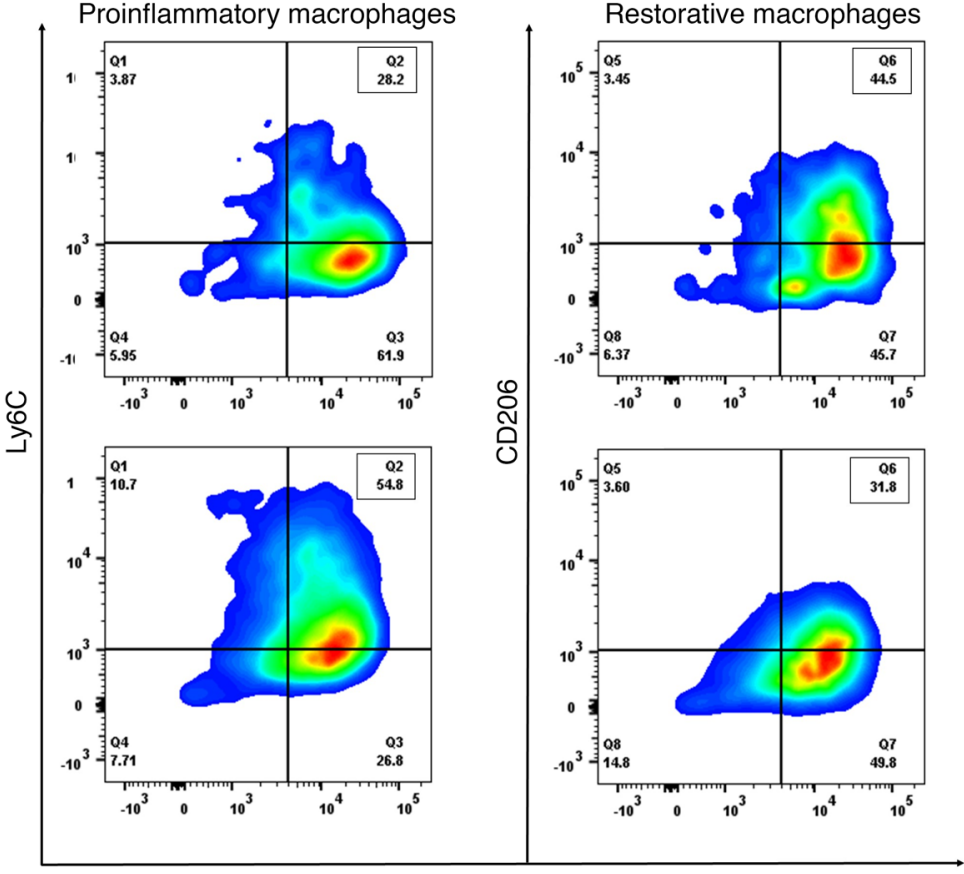

$\mathrm{F} 4 / 80$
Figure 8. Deletion of miR-223 impairs alternative activation of hepatic macrophages in SRLI. (A) Representative FACS analysis of primary hepatic macrophages from mice from the experiment in Figure 4B showing single CD11b-positive (total macrophages) and double Ly6C- and F4/80-positive (proinflammatory macrophages) or CD206- and F4/80-positive (restorative macrophages) cells. Upper quadrants on the CD11b axis and upper right quadrant on the Ly6C and F4/80 or CD206 and F4/80 axis represent the percentage of single-positive or double-positive cells, respectively. (B) Percentage of double Ly6C- and F4/80-positive or CD206- and F4/80-positive cells. ${ }^{*} P<0.05$, ${ }^{* *} P$ $<0.001,2$-way ANOVA, $n=3-4$. Results are displayed as means \pm SD. Representative experiment of 2 repeats. facturer's instructions. Female miR-223-/- mice treated for a 1-week course of CCL 4 were injected once with $5 \times 10^{6}$ isolated neutrophils via i.v. injection at day 1 of the recovery period ( 4 days after CCL4 treatment). Three days after neutrophil injection, the mice were euthanized to harvest liver tissue.

Histology and IHC. Four-micrometer-thick formalin-fixed liver sections were preincubated with $3 \%$ hydrogen peroxide for 10 minutes and blocked with 5\% BSA at room temperature (RT) for 2 hours. Primary rat monoclonal anti-Ly6C (1:100 dilution; catalog ab15627, Abcam) or anti-F4/80 (1:50; catalog MCA497GA, Bio-Rad) and rabbit monoclonal anti-CD163 (1:500; catalog ab182422, Abcam) or polyclonal antiCCR5 (1:100; catalog PA5-78949, Invitrogen), aSMA (1:500; catalog ab124964, Abcam), or polyclonal anti-CD14 (1:100; catalog sc-9150, Santa Cruz Biotechnology) antibodies were incubated on the blocked sections at $4^{\circ} \mathrm{C}$ in Dako (Odense, Denmark) Antibody Diluent with
Background Reducing Components, except anti-Ly6C antibody that was diluted in PBS containing 1\% BSA plus 1\% FBS. With the exception of anti-F4/80 antibody that required antigen retrieval in TBS-T with $2 \%$ BSA plus 1\% Triton X-100 for 30 minutes at RT, the antibodies were antigen-retrieved in boiling citrate buffer (except for anti-CCR5 antibody, pH 9) for 20 minutes. Permeabilization with $0.2 \%$ Tween-20 in PBS for 30 minutes was required before the blocking step in the sections to be incubated with anti-Ly6C antibody. After overnight incubation with primary antibody, the sections were washed in TBS-Tween and incubated with ready-to-use HRP-linked anti-rat or anti-rabbit secondary IgG antibody (ImmPRESS HRP reagents, Vector Laboratories) for 1 hour at RT. Color was developed with DAB solution (Vector Laboratories) for up to 10 seconds. Nuclei were then counterstained with Mayer's hematoxylin (Sigma-Aldrich) for 2 minutes, followed by dehydration and embedding in glycerol. F4/80-, Ly6C-, CD163-, CD14-, or 
A

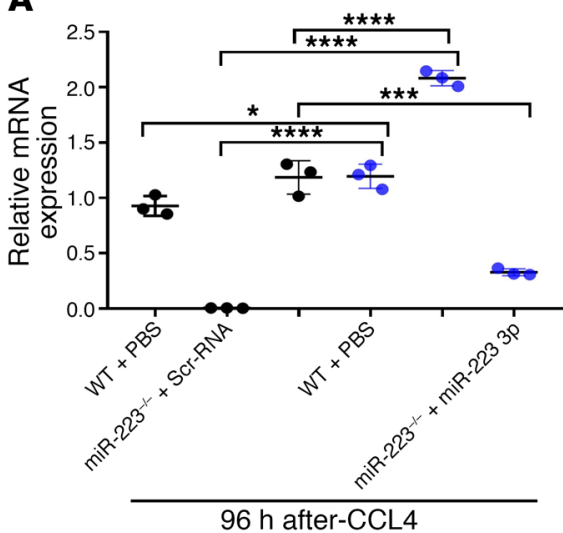

C
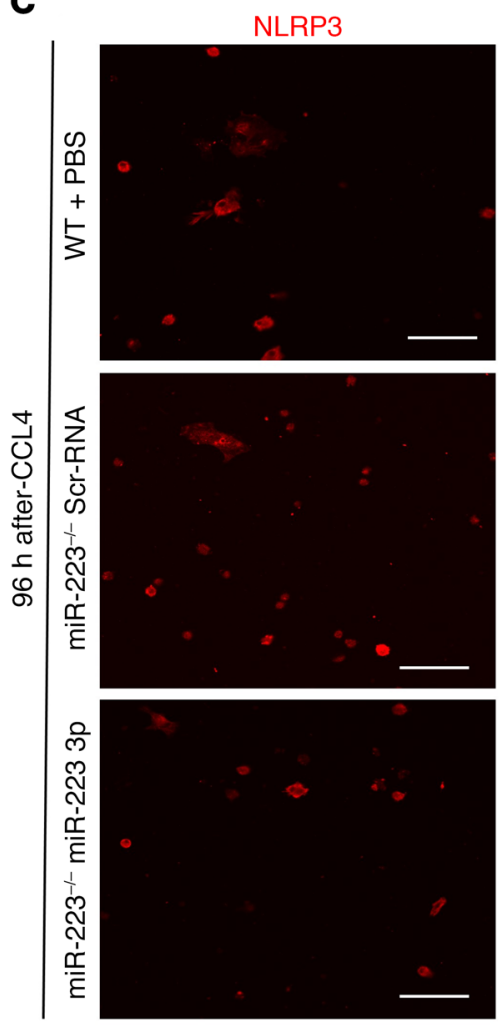

D

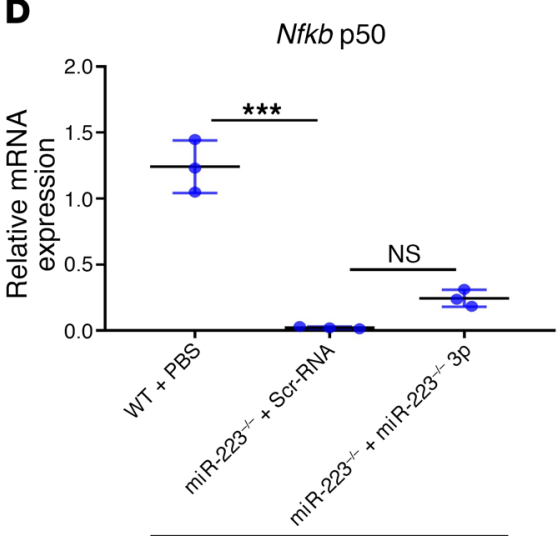

$96 \mathrm{~h}$ after-CCL4
B

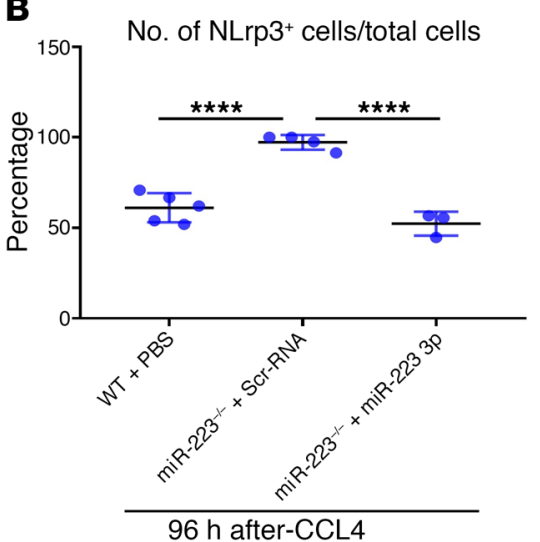

DAPI
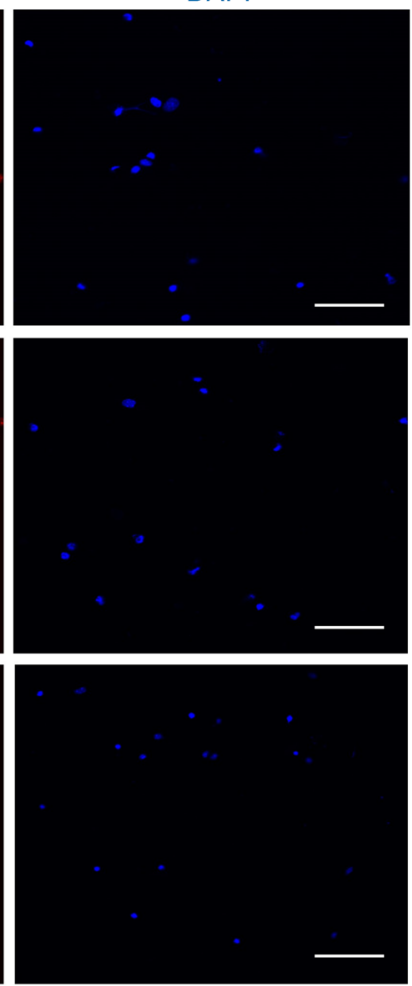

E

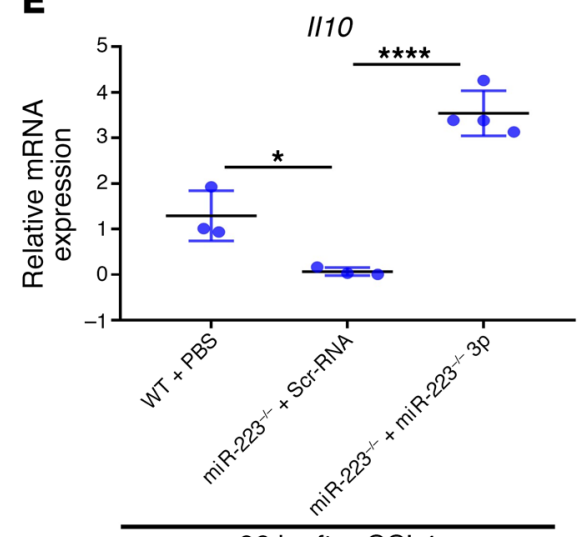

$96 \mathrm{~h}$ after-CCL4
Figure 9. miR-223 abolition augments NLRP3 expression in hepatic macrophages during SRLI. Macrophages were isolated from mouse livers from the experiment in Figure 5B. (A) Expression of miR-223 3p and Nlrp3 transcripts normalized by $B 2 m$ mRNA and measured by real-time quantitative RT-PCR. ${ }^{*} P<0.05$, ${ }^{* *} P<0.001$, ${ }^{* * *} P<0.0001,2$-way ANOVA, $n=3$. (B) Number of cells positive for anti-NLRP3 mAb normalized by total cell number per field in 10 random pictures, quantified with Imagej and expressed as percentage. ${ }^{* * *} P<0.0001$, 1-way ANOVA, $n=3-5$ per group. (C) Representative confocal immunofluorescent images of NLRP3-expressing cells (red) and nuclei (blue) stained with anti-NLRP3 mAb or DAPI, respectively. Individual merges are shown where indicated. For clear distinction of area positive for anti-NLRP3 $\mathrm{mAb}$, white arrowheads indicate cells and cellular areas negative for anti-NLRP3 mAb. Scale bars: 100 $\mu \mathrm{m}$. (D and $\mathbf{E}$ ) Expression of $N f k b$ p50 and I/10 mRNA normalized by $B 2 m$ housekeeping gene and quantified by quantitative RT-PCR. ${ }^{*} P<$ 0.05 , ${ }^{* *} P<0.001,{ }^{* * *} P<0.0001$, 1-way ANOVA, $n=3-4$ per group. Data are shown as means \pm SD. 


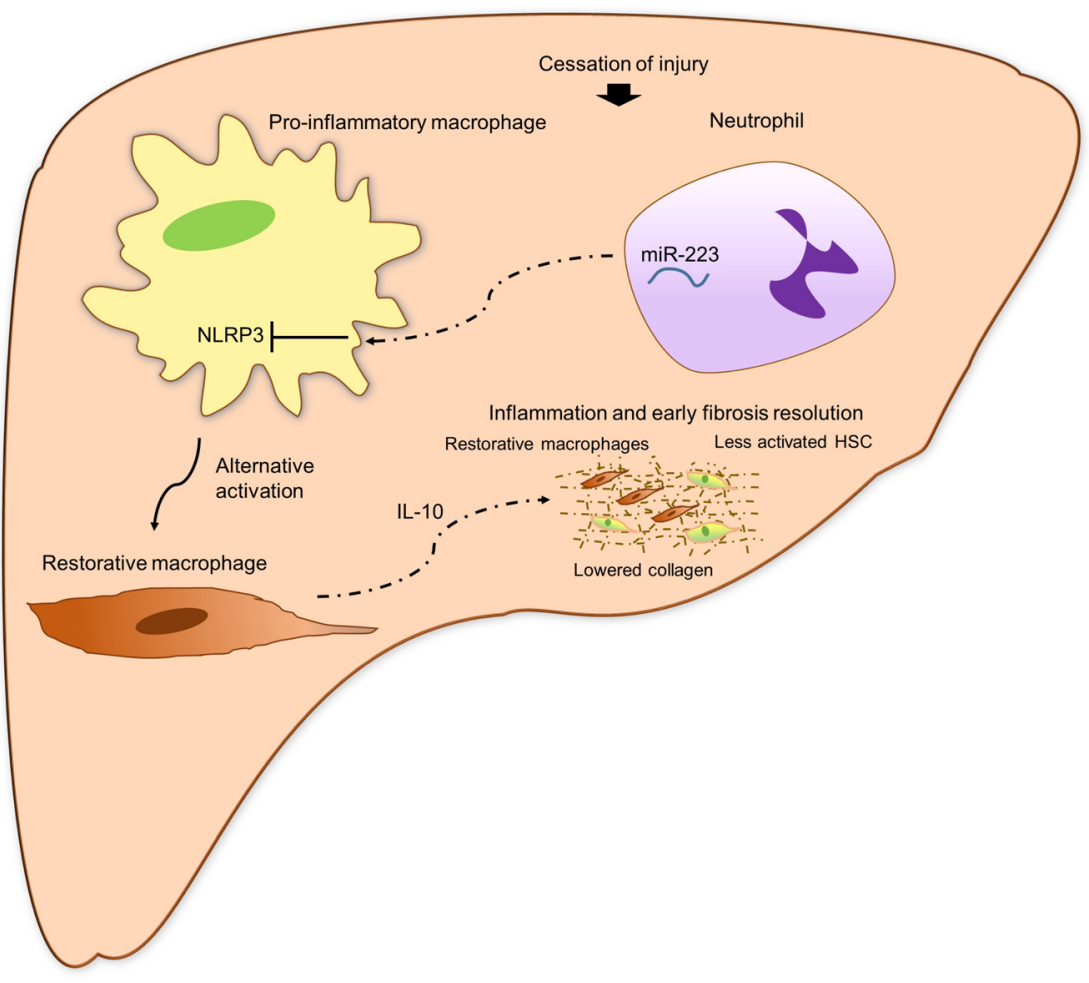

Figure 10. Schematic representation of the potential resolutive mechanism of neutrophils during SRLI. In the liver, neutrophils mediate the silencing of NLRP3 in proinflammatory macrophages via miR-223 and induce their alternative activation into a restorative phenotype after the cessation of injury. Then, restorative macrophages release IL-10 that indirectly resolves inflammation and early fibrosis by reducing the activation of HSCs and ameliorating de novo collagen formation and deposition.

aSMA-positive cells were quantified in 10 randomly selected fields (×200 magnification) imaged using a Nanozoomer 2.0HT Slide Scanner microscope (Hamamatsu Photonics K.K.). The area occupied was analyzed by selection of brown areas using an unchanged threshold value in the macro function of ImageJ software (NIH). Results were represented as percentage of area occupied by positive cells per total area in each specimen.

To identify collagen accumulation in liver tissue, formalin-fixed hepatic sections of $4 \mu \mathrm{m}$ thickness were stained for 2 hours at RT with an aqueous solution of saturated picric acid (Sigma-Aldrich) mixed with $0.1 \%$ Fast Green FCF and 0.1\% Direct Red Dye (Sigma-Aldrich). Ten randomly selected fields ( $\times 100$ magnification) were imaged and photographed as above, and the percentage of Sirius red-stained area was measured by ImageJ software with adjusted, unchanged threshold setting using macro function. Results were expressed as percentage of area occupied by Sirius red-stained fibrils.

Immunofluorescence in primary hepatic macrophages. $75 \times 10^{3}$ cells were plated overnight on a Nunc Lab-Tek II 4-well chamber slide (Thermo Fisher Scientific) to allow attachment and differentiation. After $18-20$ hours of incubation at $37^{\circ} \mathrm{C}, 5 \% \mathrm{CO}_{2}$, and $85 \%-98 \%$ humidity, the cells were washed and fixed with $4 \%$ paraformaldehyde for 10 minutes at RT. Next, the cells were washed, permeabilized (0.2\% Tween-20 in PBS, 30 minutes), and incubated at $4^{\circ} \mathrm{C}$ with mouse monoclonal anti-NLRP3 (1:100; catalog AG-20B-0014-C100, AdipoGen) or rabbit polyclonal anti-CD177 (1:50; catalog ab203025, Abcam) antibody diluted in Dako Antibody Diluent with Background Reducing Components. After overnight incubation, the cells were washed and treated with Alexa Fluor 488 anti-rabbit or Alexa Fluor 598 anti-mouse IgG antibody (1:1000; Invitrogen) for 1 hour at RT in the dark, followed by washing and 5 minutes of nuclei staining with DAPI diluted at 1:200 in PBS. The chamber was then removed, and the cells were mounted on a cover glass with Dako Fluorescent Mounting Media and analyzed with an Olympus FV1000 Confocal Microscope (Shinjuku) by repeat $x-y$ scanning at 12.5 resolution pixels and unchanged laser power. Results are expressed as percentage of anti-NLRP3-positive cells or as a ratio of anti-CD177-positive areas per total number of cells per field, analyzed by macro function of Image J and normalized by total number of nuclear cells in each field (×450 magnification).

Serum ALT and cytokine measurements. Mouse serum collected at RT from blood coagulated for 2 hours was subjected to alanine aminotransferase (ALT) analysis according to the manufacturer's instructions (Infinity ALT, Thermo Fisher Scientific).

Serum IL-12. Mouse IL-12 was measured in $13 \mu \mathrm{L}$ of diluted serum (1:5) with biotin-conjugated anti-mouse IL-12 mAb, following the manufacturer's instructions (Mouse IL-12 ELISA kit, Invitrogen).

Serum IL-10. Mouse IL-10 was quantified in $13 \mu \mathrm{L}$ diluted serum (1:4) according to the manufacturer's instructions (Mouse IL-10 ELISA kit, Invitrogen), except that TMB substrate addition was replaced by incubation with chemiluminescent SuperSignal ELISA Femto (1:1 dilution; Thermo Fisher Scientific) for 1-5 minutes in the dark. Results were calculated from luminescence units in linear regression standard curve.

Blood neutrophil counting. Mouse blood smears collected from the tail vein on glass slides were allowed to dry briefly, and immediately fixed in pure methanol (Sigma-Aldrich) at RT. After fixation for $30 \mathrm{sec}-$ onds, the smears were washed gently with tap water, dried overnight, and stained with 1:11 diluted Giemsa (Ricca Chemical) in Giemsa buffer (Sigma-Aldrich) for 45 minutes at RT.

Real time RT-PCR. Total RNA was isolated with TRIzol Reagent (Sigma-Aldrich). One microgram of total RNA was reverse-transcribed into cDNA using the iScript cDNA Synthesis Kit (Bio-Rad). The fol- 
lowing mouse SYBR Green primers were purchased from Integrated DNA Technologies (ITD): Ager forward 5'-TCACAGAAACCGGCGATGA-3', reverse 5'-CGATCTGGGTGCTCTTACGG-3'; Arg1 forward 5'-CTCCAAGCCAAAGTCCTTAGAG-3', reverse 5'-AGGAGCTGTCATTAGGGACATC-3'; B2m forward CCCCACTGAGACTGATACATACG, reverse CGATCCCAGTAGACGGTCTTG; Ccl2 forward 5'-TTAAAAACCTGGATCGGAACCAA-3', reverse 5'-GCATTAGCTTCAGATTTACGGGT-3'; Ccl4 forward 5'-TTCCTGCTGTTTCTCTTACACC-3', reverse 5'-CTGTCTGCCTCTTTTGGTCAG-3'; Il10 forward 5'-GCTCTTACTGACTGGCATGAG-3', reverse 5'-CGCAGCTCTAGGAGCATGTG-3'; Il12 p35 forward 5'-GAGGACTTGAAGATGTACCAG-3'， reverse 5'-TTCTATCTGTGTGAGGAGGGC-3'; iNos forward 5'-CAGCTGGGCTGTACAAACCTT-3', reverse 5'-CATTGGAAGTGAAGCGGTTCG-3'; Mmp3 forward 5'-ACATGGAGACTTTGTCCCTTTTG-3', reverse 5'-TTGGCTGAGTGGTAGAGTCCC-3'; Mmp8 forward 5'-CCTTGCCCATGCCTTTCAAC-3', reverse 5'-TCATGAGCAGCCACGAGAAA-3'; $N f k b$ p50 forward 5'-GGTCACCCATGGCACCATAA-3', reverse 5'-AGCTGCAGAGCCTTCTCAAG-3'; Nlrp3 forward 5'-ATTACCCGCCCGAGAAAGG-3', reverse 5'-TCGCAGCAAAGATCCACACAG-3'.

$B 2 m$ was used to normalize data and to control for RNA integrity. cDNA amplification reactions were performed using a CFX96 realtime system (Bio-Rad).

Specific cDNA from miR-223 3p or U6 was transcribed from 10 ng total RNA by reverse transcriptase reaction with the TaqMan MicroRNA Reverse Transcription kit (Applied Biosystems). TaqMan MicroRNA Assay kit specific for mmu-miR-223 3p and mmu-U6 was used for amplification reactions (Applied Biosystems). U6 served as a housekeeping gene. Reactions were performed in a Step One Plus realtime amplification system (Applied Biosystems).

TaqMan and SYBR Green reaction mixtures were purchased from Applied Biosystems.

Gene expression data were calculated by $\Delta \Delta \mathrm{CT}$.

Isolation of liver macrophages. Multiple mouse livers were separately digested in situ by perfusion through the infrahepatic vein cave with EGTA, pronase, and collagenase D solutions subsequently as previously described (58). Then, each liver was placed individually in ice-cooled enzyme buffer solution (prepared as previously described, ref. 58) on ice and transferred to room temperature for 30 minutes immediately before in vitro digestion with pronase and collagenase D solution (containing 1\% DNAse) on a stirring plate for 24 minutes at $40^{\circ} \mathrm{C}$ as described elsewhere (58). Next, debris filtration and total liver cell precipitation were performed as previously described (58). Parenchymal and nonparenchymal cells were gradient-separated in 2 layers with $37.5 \%$ Nycodenz solution (Axis-Shield) as previously shown (58). The nonparenchymal cell fraction was collected from the upper layer after centrifugation at $580 \mathrm{~g}$ for 10 minutes at $4^{\circ} \mathrm{C}$. The collected cell layer was then resuspended in MACS buffer (PBS with 2 mM EDTA and 2\% FBS) and incubated with rat biotinylated F4/80 $\mathrm{mAb}\left(1: 100\right.$; catalog 123105 , BioLegend) at $1.25 \mathrm{~g}$ per $10^{6}$ cells for 30 minutes on ice. Afterward, the cells were washed in MACS buffer, centrifuged at $700 \mathrm{~g}$ for 5 minutes at RT, resuspended in MACS buffer, and incubated with $9 \mu \mathrm{L}$ of anti-biotin microbeads (Miltenyi Biotec) per $10^{6}$ cells for 1 hour at $4^{\circ} \mathrm{C}$ in the dark. Final macrophage collection was achieved with MACS magnetic cell separation columns (Miltenyi Biotec) according to the manufacturer's instructions. For immunofluorescence and quantitative RT-PCR analysis, the purity of the final isolated macrophages was enhanced by plating of the cells on a T25 tissue culture flask with the macrophage/monocyte-specific culture medium RPMI 1640 (Gibco) containing 10\% FBS and 1\% antibiotic/ antimycotic to get a readout of possible unwanted nonparenchymal cells (stellate and endothelial cells). Cells were allowed to attach and differentiate on plastic surface overnight in an incubator at $37^{\circ} \mathrm{C}, 5 \%$ $\mathrm{CO}_{2}$, and $85 \%-98 \%$ humidity. About $1 \times 10^{6}$ to $4 \times 10^{6}$ macrophages were isolated per liver by this method.

FACS analysis. Total liver macrophages resuspended in MACS buffer were fixed in $2 \%$ paraformaldehyde for 10 minutes at RT. After overnight storage at $4^{\circ} \mathrm{C}$, the cells were washed, centrifuged, and blocked with rat anti-CD16/32 mAb (1:500; catalog 14-0161-82, Thermo Fisher Scientific) at RT for 15 minutes. Staining of total macrophages was then followed with $2 \mathrm{~g}$ of APC-anti-biotin streptavidin (BioLegend) at $4^{\circ} \mathrm{C}$ in the dark. After 20 minutes, rat FITC-anti-CD11b (1:400; catalog 101205, BioLegend), rat Brilliant Violet 421 -anti-Ly6C $\left(2.5 \mu \mathrm{L}\right.$ per $0.5 \times 10^{6}$ cells; catalog 128031, BioLegend), and rat PerCP-Cy5.5-anti-CD206 (1:100; catalog 141715, BioLegend) mAbs were incubated with the cells for 30 minutes at $4^{\circ} \mathrm{C}$ in the dark. The cells were finally centrifuged, washed, and resuspended in MACS buffer. Subsets of total macrophages were then identified with a FACSCanto RUO-ORANGE analyzer (BD Biosciences) and represented as percentage of double F4/80- and Ly6C- or CD206-double positive cells at unchanged $x, y$ values.

Immunoblot analysis. Twenty $\mu \mathrm{g}$ of total tissue protein were loaded in $5 \% \beta$-mercaptoethanol-containing SDS, boiled for 5 minutes, and electrophoresed via an Any $\mathrm{kD}$ precast Tris-Glycine gel (Bio-Rad) at $60 \mathrm{~V}$ for 15 minutes, followed by $100 \mathrm{~V}$ until the end of the run. TrisGlycine-SDS at pH 8.3 was used as a running buffer. After electrophoresis, the proteins were transferred from the gel to a nitrocellulose membrane in a Trans Blot Turbo Transfer system (Bio-Rad) for 7 minutes at $25 \mathrm{~V}$. An ethanol-based solution was used as transfer buffer (Bio-Rad). Once proteins were transferred, blocking of the membrane was proceeded by 1 hour in TBS-Tween-diluted 5\% dried milk. The membrane was then incubated with mouse anti-CCL2 (1:000; catalog 2029, Cell Signaling Technology). After overnight incubation with primary antibody, the membrane was washed 3 times in TBS-Tween and incubated with HRP-linked anti-mouse antibodies for 1 hour at RT. Finally, protein bands were visualized with an enhanced chemiluminescence reagent (Pico or Femto, Thermo Fisher Scientific) and digitized in a CCD camera (ChemiDoc, Bio-Rad). Intensity of bands was quantified with ImageJ. Results are expressed as arbitrary units of protein expression normalized against the housekeeping protein ACTB housekeeping protein.

Statistics. Shapiro-Wilk test $(\alpha=0.05)$ was used to check normal Gaussian distribution (Supplemental Figure 18). Significance of 2-group comparisons was determined with 2-tailed Student's $t$ test. Significance of more than 2 groups was analyzed by 1-way ANOVA. Significance of multiple comparisons between 2 or more groups was measured with 2-way ANOVA. Tukey's post hoc test was used to correct both 1- and 2-way ANOVA. $P$ values less than 0.05 were considered significant. Error bars represent means \pm SD. Experiments were repeated at least 2 or 3 times, and assays were performed in duplicate or triplicate.

Study approval. The experimental protocols were approved by the IACUC of UCSD under protocol $\$ 11200$.

\section{Author contributions}

CJC partially conceived the idea, designed and performed experiments, analyzed data, and wrote the manuscript. MT 
designed and performed experiments and analyzed data. CDJ, HDP, YCL, CLL, and JB provided technical help. NA and XDMDJ supplied the miR-223 3p and Invivofectamine 3. AEF conceived the idea, helped design the experiments, provided the funding for the study, and helped draft and critically revised the manuscript.

\section{Acknowledgments}

This study was supported by the NIH (R01 DK113592 and U01 AA024206 to AEF), the microscopy core facility of the Depart- ment of Neuroscience at UCSD (grant NS047101), the Flow Cytometry Core at the San Diego Core Center for AIDS Research (P30 AI036214), the VA San Diego Healthcare System, and the San Diego Veterans Medical Research Foundation.

Address correspondence to: Ariel E. Feldstein, Division of Pediatric Gastroenterology, Hepatology, and Nutrition, UCSD School of Medicine, 3020 Children's Way, MC 5030, San Diego, California 92103-8450, USA. Phone: 858.966.8907; Email: afeldstein@ ucsd.edu.
1. Sun B, Karin M. Obesity, inflammation, and liver cancer. J Hepatol. 2012;56(3):704-713.

2. Robinson MW, Harmon C, O'Farrelly C. Liver immunology and its role in inflammation and homeostasis. Cell Mol Immunol. 2016;13(3):267-276.

3. Medzhitov R. Origin and physiological roles of inflammation. Nature. 2008;454(7203):428-435.

4. Liaskou E, Wilson DV, Oo YH. Innate immune cells in liver inflammation. Mediators Inflamm. 2012;2012:949157.

5. Koyama Y, Brenner DA. Liver inflammation and fibrosis. J Clin Invest. 2017;127(1):55-64.

6. Czaja AJ. Hepatic inflammation and progressive liver fibrosis in chronic liver disease. World J Gastroenterol. 2014;20(10):2515-2532.

7. Arthur MJ. Reversibility of liver fibrosis and cirrhosis following treatment for hepatitis C. Gastroenterology. 2002;122(5):1525-1528.

8. Czaja AJ, Carpenter HA. Decreased fibrosis during corticosteroid therapy of autoimmune hepatitis. J Hepatol. 2004;40(4):646-652.

9. Dufour JF, DeLellis R, Kaplan MM. Reversibility of hepatic fibrosis in autoimmune hepatitis. Ann Intern Med. 1997;127(11):981-985.

10. Lassailly $G$, et al. Bariatric surgery reduces features of nonalcoholic steatohepatitis in morbidly obese patients. Gastroenterology. 2015;149(2):379-388.

11. Dixon JB, Bhathal PS, Hughes NR, O'Brien PE. Nonalcoholic fatty liver disease: improvement in liver histological analysis with weight loss. Hepatology. 2004;39(6):1647-1654.

12. Ellis EL, Mann DA. Clinical evidence for the regression of liver fibrosis. J Hepatol. 2012;56(5):1171-1180.

13. Horckmans M, et al. Neutrophils orchestrate post-myocardial infarction healing by polarizing macrophages towards a reparative phenotype. Eur Heart J. 2017;38(3):187-197.

14. Nishio N, Okawa Y, Sakurai H, Isobe K. Neutrophil depletion delays wound repair in aged mice. Age (Dordr). 2008;30(1):11-19.

15. Cumpelik A, Ankli B, Zecher D, Schifferli JA. Neutrophil microvesicles resolve gout by inhibiting C5a-mediated priming of the inflammasome. Ann Rheum Dis. 2016;75(6):1236-1245.

16. Headland SE, et al. Neutrophil-derived microvesicles enter cartilage and protect the joint in inflammatory arthritis. Sci Transl Med. 2015;7(315):315ra190.

17. Kubes P. The enigmatic neutrophil: what we do not know. Cell Tissue Res. 2018;371(3):399-406. 18. Bauernfeind F, Rieger A, Schildberg FA, Knolle
PA, Schmid-Burgk JL, Hornung V. NLRP3 inflammasome activity is negatively controlled by miR-223. J Immunol. 2012;189(8):4175-4181.

19. Johnnidis JB, et al. Regulation of progenitor cell proliferation and granulocyte function by microRNA-223. Nature. 2008;451(7182):1125-1129.

20. Neudecker V, et al. Myeloid-derived miR-223 regulates intestinal inflammation via repression of the NLRP3 inflammasome. J Exp Med. 2017;214(6):1737-1752.

21. Cheung O, et al. Nonalcoholic steatohepatitis is associated with altered hepatic MicroRNA expression. Hepatology. 2008;48(6):1810-1820.

22. Ramachandran P, et al. Differential Ly-6C expression identifies the recruited macrophage phenotype, which orchestrates the regression of murine liver fibrosis. Proc Natl Acad Sci U S A. 2012;109(46):E3186-E3195.

23. Ishak $\mathrm{K}$, et al. Histological grading and staging of chronic hepatitis. J Hepatol. 1995;22(6):696-699.

24. Jablonski KA, et al. Novel markers to delineate murine M1 and M2 macrophages. PLoS One. 2015;10(12):e0145342.

25. Bouhlel MA, et al. PPARgamma activation primes human monocytes into alternative M2 macrophages with anti-inflammatory properties. Cel Metab. 2007;6(2):137-143.

26. Sierra-Filardi E, Vega MA, Sánchez-Mateos $P$, Corbí AL, Puig-Kröger A. Heme oxygenase-1 expression in M-CSF-polarized M2 macrophages contributes to LPS-induced IL-10 release. Immunobiology. 2010;215(9-10):788-795.

27. Liu C, et al. Targeting the shift from M1 to M2 macrophages in experimental autoimmune encephalomyelitis mice treated with fasudil. PLoS One. 2013;8(2):e54841.

28. Sanyal R, et al. MS4A4A: a novel cell surface marker for M2 macrophages and plasma cells. Immunol Cell Biol. 2017;95(7):611-619.

29. Martinez FO, Gordon S, Locati M, Mantovani A. Transcriptional profiling of the human monocyte-to-macrophage differentiation and polarization: new molecules and patterns of gene expression. J Immunol. 2006;177(10):7303-7311.

30. Deshmane SL, Kremlev S, Amini S, Sawaya BE. Monocyte chemoattractant protein-1 (MCP-1): an overview. Interferon Cytokine Res. 2009;29(6):313-326.

31. Mantovani A, Sica A, Sozzani S, Allavena P, Vecchi A, Locati M. The chemokine system in diverse forms of macrophage activation and polarization. Trends Immunol. 2004;25(12):677-686.

32. Sica A, Mantovani A. Macrophage plasticity and polarization: in vivo veritas. JClin Invest.
2012;122(3):787-795.

33. Gordon S, Martinez FO. Alternative activation of macrophages: mechanism and functions. Immunity. 2010;32(5):593-604.

34. Lolmede $\mathrm{K}$, et al. Inflammatory and alternatively activated human macrophages attract vesselassociated stem cells, relying on separate HMGB1- and MMP-9-dependent pathways. JLeukoc Biol. 2009;85(5):779-787.

35. Sachs UJ, et al. The neutrophil-specific antigen CD177 is a counter-receptor for platelet endothelial cell adhesion molecule-1 (CD31). J Biol Chem. 2007;282(32):23603-23612.

36. Kissel K, Santoso S, Hofmann C, Stroncek D, Bux J. Molecular basis of the neutrophil glycoprotein NB1 (CD177) involved in the pathogenesis of immune neutropenias and transfusion reactions. Eur J Immunol. 2001;31(5):1301-1309.

37. Bauer S, Abdgawad M, Gunnarsson L, Segelmark M, Tapper H, Hellmark T. Proteinase 3 and CD177 are expressed on the plasma membrane of the same subset of neutrophils. J Leukoc Biol. 2007;81(2):458-464.

38. Timár CI, et al. Antibacterial effect of microvesicles released from human neutrophilic granulocytes. Blood. 2013;121(3):510-518.

39. Arrese M, Eguchi A, Feldstein AE. Circulating microRNAs: emerging biomarkers of liver disease. Semin Liver Dis. 2015;35(1):43-54.

40. Freitas-Lopes MA, Mafra K, David BA, Carvalho-Gontijo R, Menezes GB. Differential location and distribution of hepatic immune cells. Cells. 2017;6(4):E48

41. Gong JP, et al. Liver sinusoidal endothelial cell injury by neutrophils in rats with acute obstructive cholangitis. World J Gastroenterol. 2002;8(2):342-345

42. Krenkel O, Tacke F. Liver macrophages in tissue homeostasis and disease. Nat Rev Immunol. 2017;17(5):306-321.

43. Racanelli V, Rehermann B. The liver as an immunological organ. Hepatology. 2006;43(2 suppl 1):S54-S62.

44. Wessells J, et al. BCL-3 and NF-kappaB p50 attenuate lipopolysaccharide-induced inflammatory responses in macrophages. J Biol Chem. 2004;279(48):49995-50003.

45. Cao S, Zhang X, Edwards JP, Mosser DM. NF-kappaB1 (p50) homodimers differentially regulate pro- and anti-inflammatory cytokines in macrophages. JBiol Chem. 2006;281(36):26041-26050.

46. Weiss U. Inflammation. Nature. 2008;454(7203):427.

47. Wynn TA, Ramalingam TR. Mechanisms of fibro- 
sis: therapeutic translation for fibrotic disease. Nat Med. 2012;18(7):1028-1040.

48. Wynn TA, Vannella KM. Macrophages in tissue repair, regeneration, and fibrosis. Immunity. 2016;44(3):450-462.

49. Kohli P, Levy BD. Resolvins and protectins: mediating solutions to inflammation. Br JPharmacol. 2009;158(4):960-971.

50. Serhan CN, Yacoubian S, Yang R. Anti-inflammatory and proresolving lipid mediators. Annu Rev Pathol. 2008;3:279-312.

51. Serhan CN, et al. Maresins: novel macrophage mediators with potent antiinflammatory and proresolving actions. J Exp Med. 2009;206(1):15-23.

52. Uhl B, et al. Aged neutrophils contribute to the first line of defense in the acute inflammatory response. Blood. 2016;128(19):2327-2337.

53. Brunt EM. Histological assessment of nonalcoholic fatty liver disease in adults and children. Clin Liver Dis (Hoboken). 2012;1(4):108-111.

54. Theise ND. Histopathology of alcoholic liver disease. Clin Liver Dis (Hoboken). 2013;2(2):64-67.

55. Altamirano J, et al. A histologic scoring system for prognosis of patients with alcoholic hepatitis.
Gastroenterology. 2014;146(5):1231-1239.e1.

56. Latz E, Xiao TS, Stutz A. Activation and regulation of the inflammasomes. Nat Rev Immunol. 2013;13(6):397-411.

57. Zhuang G, et al. A novel regulator of macrophage activation: MiR-223 in obesity-associated adipose tissue inflammation. Circulation. 2012;125(23):2892-2903.

58. Mederacke I, Dapito DH, Affò S, Uchinami H, Schwabe RF. High-yield and high-purity isolation of hepatic stellate cells from normal and fibrotic mouse livers. Nat Protoc. 2015;10(2):305-315. 